\title{
Perspective on photonic memristive neuromorphic computing
}

\author{
Elena Goi ${ }^{1,2}$, Qiming Zhang ${ }^{1,2}$, Xi Chen ${ }^{1,2}$, Haitao Luan ${ }^{1,2}$ and Min Gu ${ }^{1,2^{*}}$
}

\footnotetext{
* Correspondence: gumin@usst.edu. $\mathrm{cn}$

${ }^{1}$ Laboratory of Artificial-Intelligence Nanophotonics, School of Science, RMIT University, Melbourne, Victoria 3001, Australia

${ }^{2}$ Centre for Artificial-Intelligence Nanophotonics, School of Optical-Electrical and Computer Engineering, University of Shanghai for Science and Technology, Shanghai 200093, China
}

\begin{abstract}
Neuromorphic computing applies concepts extracted from neuroscience to develop devices shaped like neural systems and achieve brain-like capacity and efficiency. In this way, neuromorphic machines, able to learn from the surrounding environment to deduce abstract concepts and to make decisions, promise to start a technological revolution transforming our society and our life. Current electronic implementations of neuromorphic architectures are still far from competing with their biological counterparts in terms of real-time information-processing capabilities, packing density and energy efficiency. A solution to this impasse is represented by the application of photonic principles to the neuromorphic domain creating in this way the field of neuromorphic photonics. This new field combines the advantages of photonics and neuromorphic architectures to build systems with high efficiency, high interconnectivity and high information density, and paves the way to ultrafast, power efficient and low cost and complex signal processing. In this Perspective, we review the rapid development of the neuromorphic computing field both in the electronic and in the photonic domain focusing on the role and the applications of memristors. We discuss the need and the possibility to conceive a photonic memristor and we offer a positive outlook on the challenges and opportunities for the ambitious goal of realising the next generation of full-optical neuromorphic hardware.
\end{abstract}

Keywords: Neuromorphic photonics, Neuromorphic computing, Memristors, Photonic memristors, Artificial intelligence, Graphene oxide

\section{Introduction}

The ability to achieve artificial intelligence (AI) reproducing human reasoning in machines has been an ambition of scientific research since the first formal design of McCulloch and Pitts's neuron in 1943 [1]. Over the years, AI has been implemented in several different ways leading to increasingly advanced applications. The first AI generation consisted in rules-based algorithms that emulated classic logic to draw reasoned conclusions within pertinent and specific problems, while the second generation used deep-learning networks to analyse and manipulate audio and video content, focusing more and more on sensing and perception [2]. The third generation, based on big data and cloud computing, is currently developing and will extend AI ambit to autonomous decision, deduction, adaption and interpretation, emulating more realistically the human cognition [3].

(c) The Author(s). 2020 Open Access This article is distributed under the terms of the Creative Commons Attribution 4.0 International License (http://creativecommons.org/licenses/by/4.0/), which permits unrestricted use, distribution, and reproduction in any medium, provided you give appropriate credit to the original author(s) and the source, provide a link to the Creative Commons license, and indicate if changes were made. 
While a large part of the current AI research focuses on simulating and mimicking brain functions, neuromorphic computing aims to develop systems that take the form of the brain and whose functions are analogous to parts of the neural system. Neuromorphic systems are not necessary brain-shaped, yet they do fulfil the roles of their organic counterparts, achieving brain-like capacity and efficiency by applying concepts extracted from neuroscience. In this way, neuromorphic machines able to learn from the surrounding environment, to deduce abstract concepts and to make decisions, promise to start a technological revolution transforming our society and our life [4].

In this Perspective, we review the reasons of the rapid growth of neuromorphic computing both in the electronic and in the photonic domain, and we briefly summarise the recent advancements in these fields. Specifically, we focus on the role of memristors and their application in electronic artificial synapses and neurons, and we discuss the need and the possibility to conceive a photonic counterpart, a photonic memristor. We propose materials and suggest pathways for the realisation of photonic memristors. Consequently, we discuss several approaches for fabricating these materials to be integrated in photonic neuromorphic components. Lastly, we offer a positive outlook on the challenges and opportunities for the ambitious goal of realising the next generation of full-optical neuromorphic hardware.

\section{From von Neuman to neuromorphic computing}

In the past half century, we have witnessed a rapid evolution of digital computers due to their ability of solving structured mathematical problems. Most of the computers currently in use are designed to perform Boolean algebra and arithmetic according to the von Neumann approach that physically separates the central processing unit (CPU) and the memory (Fig. 1a). Machine instructions and data are stored in the memory and share a central communication channel, a digital bus, to the processing unit. Prespecified instructions define the procedures to operate on the data, which are transferred back and forth from the memory to the CPU where computation takes place. The limitation of modern computers is the rate at which data can be transferred between the processing unit and the memory unit, known as the memory wall [7]. No matter how fast the CPU or how vast the memory array, calculations are ultimately limited by the bandwidth of the bus connecting the two. Moreover, von Neumann computers work very efficiently when it comes to executing algorithmic instructions, but they run into trouble for highly complex or abstract computational tasks such as speech recognition or facial recognition. This happens because the continuous transfer

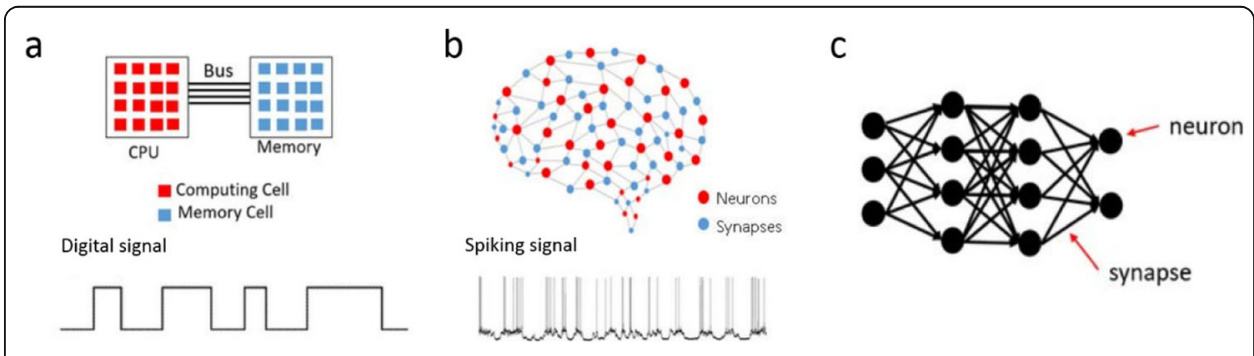

Fig. 1 Comparison of von Neumann (a), biological neural system (b) and neuromorphic computing (c). a, c Reproduce from ref. [5] with permission. b Reproduced from ref. [6] with permission 
of large amount of data between the physically separated processor and memory required for these operations in the von Neumann architecture consumes a considerable amount of energy. For example, AlphaGo, a computer program developed by Google DeepMind to play the board game Go and implementing AI on von Neuman computers, used 40 search threads, 1202 CPUs and 176 graphic processing units (GPUs) when it played against Lee Sedol [8]. It's speculated that the power consumption was above 1.2 MW [9]. For comparison, only $20 \mathrm{~W}$ can be used by the human brain, meaning that AlphaGo consumed about 60,000 [9] times more energy than Lee Sedol. Consequently, it is crucial to develop low-power consumption AI technologies for a sustainable future.

As the most powerful information processor currently known, a human brain is a system capable of ultra-fast computation with a high power-efficiency due to the presence of interconnected neural circuits that enable distributed parallel processing (Fig. 1b). Human brain has an extremely low power consumption compared with AI systems, and it is also good at learning, predicting future events, classifying objects and comprehending languages [10]. Understanding the morphology and the functionalities of the human brain led in the late '80s [11] to the development of a new computational concept called neuromorphic computing (Fig. 1c). The aim of neuromorphic computing is to build systems that mimic architecture, data processing methodology and functionalities of the biological brains and achieve the ability to analyse data sets more rapidly, more accurately, and with fewer computing resources than conventional computers. The promise of speed, intelligent performances and energy-efficiency made neuromorphic computing the perfect candidate for the next-generation computing and a very prolific research field.

\section{Neuromorphic models}

The first step towards the creation of neuromorphic computers is the understanding of neural system structures and functionalities. Biological brains have no memory elements and no central computation unit. They are highly interconnected assemblies of neurons, the computing units, and synapses, the proposed memory units, communicating by pulses of ion current mediated by neurotransmitters (Fig. 2a). The synaptic weight can be precisely adjusted by the ionic flow through two contiguous neurons and this process is believed to be responsible for the ability of biological systems to learn and function [13]. Though some sophisticated capabilities, like facial recognition, appear to be innate, human brains are largely self-programming, continuing to build associations between experiences over the course of the individual's life. Biological brains also forget: connections can decay if not reinforced. There is a degree of randomness in biological brains, too, that neuroscientists believe may be necessary for creativity and problem solving [10].

The key to high computing speed and power efficiency of biological neural systems are the small distance between the computing unit and the memory unit and the large connectivity $\left(\sim 10^{4}\right.$ connections in a mammalian cortex) between neurons [10]. Moreover, the mammalian nervous system encodes the stimulus of sensory neurons as spikes, a type of signal with both analog and digital properties, which combines analog signal efficiency and digital low noise accumulation [14].

Rather than executing a set of instructions in sequence, neurons can process data in different regions of the brain independently and in parallel [10]. In this mechanism, a given neuron receives the output signals from the neighbouring neurons through the 


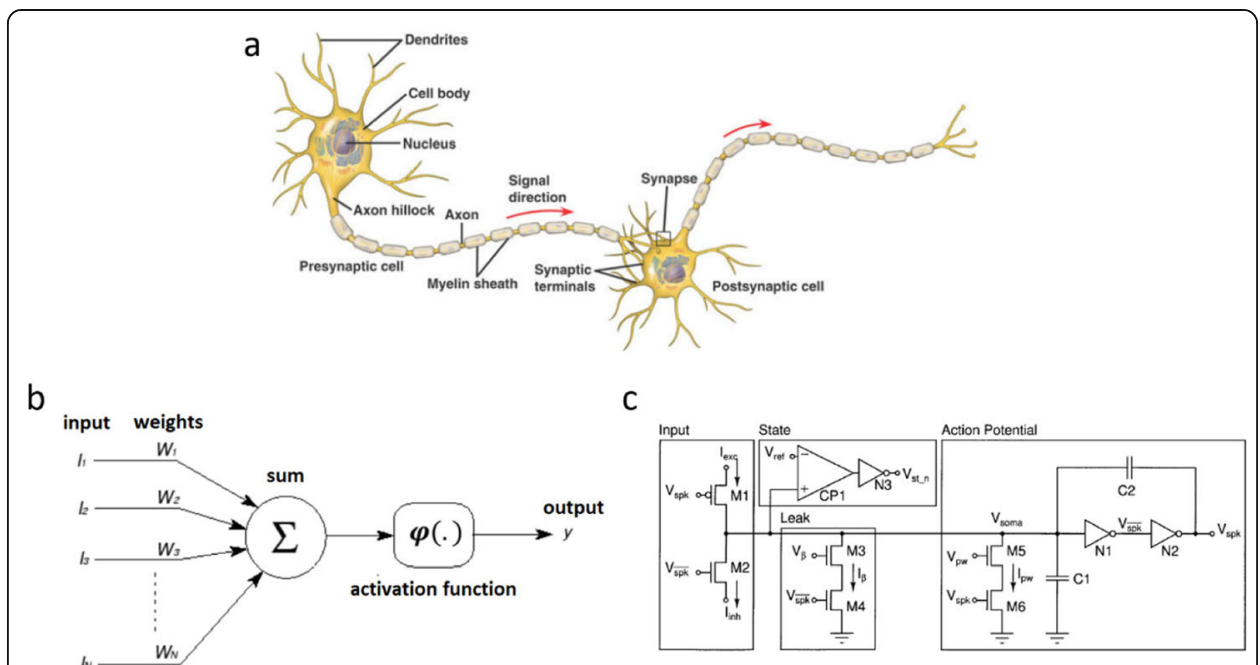

Fig. 2 a Diagram of the biological neuron highlighting the main components of the cell, the connection between axon and dendrite and the direction of signal propagation (red arrows). Reproduced with permission of@ 2005 Pearson Education, Inc. publishing as Benjamin Cummings PowerPoint Lectures for Biology, Seventh Edition Neil Campbell and Jane Reece. b Schematic illustration of McCulloch and Pitts model. The model consists of $N$ inputs $\left(I_{N}\right)$ multiplied by corresponding $N$ weights $\left(W_{N}\right)$, which are summed together $(\Sigma)$. The activation function $(\varphi(\cdot))$ evaluates the sum of the inputs and if a threshold is reached, the neuron will send its output signal. c Schematic illustration of an implementation of the leaky-integrate-and-fire (LIF) model. The four functional blocks are input, state, leak, and action potential. Combined in a circuit, they integrate linearly the input, exhibit a constant leakage, and emit a spiking signal when the potential crosses a threshold. In this way they implement an integrate-and-fire neuron. Reproduced from ref. [12] with permission

dendrites and each dendrite is able multiplicate the incoming signal for a specific weight value. Subsequently, all the weighted signals proceed to the soma that act as summation function. The result of the multiplication by the dendrites and the summation behaviour of the soma is a linear combination of all the incoming signals neurons and as such, it can be described mathematically, and the interconnected network can be represented by a matrix.

In modelling the brain, it is necessary to consider the biological mechanism by which chains of synaptic current are generated and propagated and the role of these spikes in memory and learning. A neuron, if considered as a computing unit, has two main functionalities: integrate and sum spiking signals from other neurons and generate a spiking signal whenever the integrated signal excesses the threshold voltage. Based on the deep understanding of neuron functions, several neuron models have been developed over the last century to describe mathematically the properties of the neural system and facilitate the replication of those properties in artificial architectures. The earliest models were the McCulloch and Pitts model (1943) [1] (Fig. 2b), the Hodgkin-Huxley model (1952) [15], the LIF model (1965) [16] (Fig. 2c), and many other followed with increased complexity.

All the attempts to model neural cells are useful for the development of artificial networks but in the end inaccurate, since neural computing and memory principles are not completely understood. However, it is now widely recognised and experimentally proven that memory is stored as weights of synaptic matrices, and the essence of learning is to alter such weights based on experience [10]. The ability to learn is associated to synaptic plasticity, i.e. a weight modification that reinforces or depresses the strength 
of neuronal connections, as described by Hebb with the postulate "who fire together, wire together" (Hebb, 1949) [17]. Practically, the change of synaptic weight at specific synapses means that the same input will produce different outputs before and after the learning process. The synaptic weight matrices can in principle store enormous amounts of information that can be used to transform specific input patterns (events) to specific output patterns (memory recalls) as strength of synaptic connections in neural circuits [10]. Synaptic plasticity is a broad term that can be used to describe the changes of the strengths of synaptic connections in response to experience and neuronal activity. More in general, plasticity indicates the ability of a single cell or of the entire neural system to adapt and reorganise itself in response to new to external stimuli or changes in the environment. Several plasticity mechanisms can work together at a network level, shaping the connectivity in our brain and different types of learning can emerge depending on the synaptic configuration. The most popular variation of Hebb's rule is the spike-timing dependent plasticity (STDP), where the precise timing of pre- and post-synaptic firing is critical in determining the sign of the synaptic strength change [10]. Many additional plasticity mechanisms co-exist with STDP and, in general, with long term potentiation, such as intrinsic plasticity. While synaptic plasticity controls the dynamics at the connection between two communicating neurons, intrinsic plasticity controls the nonlinear transfer function of the neuron itself. Both have a fundamental role in the cognitive processes of brains.

\section{Early works and large-scale neuromorphic systems}

The term neuromorphic has been used to identify analog, digital, mixed-mode analog/ digital very-large-scale-integration designs, and software systems that implement neural models on von Neuman computers. However, neuromorphic computing strictly referees to hardware systems that mimic neuro-biological architectures present in the nervous system and these systems only are the subject of this perspective article.

The neuromorphic computing field has its origins in the seminal work of Carver Mead at Caltech in the late 1980s that includes the publication of his book 'Analog VLSI and Neural Systems' in 1989 and the establishment of companies such as Synaptics Inc. (1986) [18], for the development of analogue circuits based on neural networks for laptop touch pads. The foundations of Mead's approach to neuromorphic engineering rest upon the analogy between the physics of transistors operating in the sub-threshold region and biological phenomena taking place in neuro-systems.

Early works in the field consisted in the development of neuromorphic techniques to investigate biological phenomena and to demonstrate a match between models and biological networks [19]. Subsequent network research included developing neuron-inspired circuits with digitally-implemented connectivity and plasticity through a real-time closed loop [20].

Following studies included multi-neuron integrate-and-fire transceiver modules [21, 22], neuromorphic vision sensors [23], silicon cochlea [24], and medium-scale neuromorphic processors such as the Reconfigurable On-Line Learning Spiking and the cxQuad chips [25]. These devices used sub-threshold analogue circuits and demonstrated spiking deep neural networks with low latency and very high-power efficiency compared with deep networks running on a conventional digital cluster machine. More recent works showed 
that complementary metal-oxide-semiconductor (CMOS)-based neuromorphic multi-core processors with one million neurons and 256 million synapses reduced the operative power consumption by a factor $10^{4}$ with respect to the conventional CMOS architectures [26], and high-operation efficiency was also demonstrated in analog circuits with LIF neurons and silicon synapses [27, 28].

These early studies encouraged further developments of very-large-scale integration technology supported by the enormous transistor density and the technologies now available: Neurogrid as part of Stanford University's Brains in Silicon program (2014), IBM's TrueNorth as part of the Defense Advanced Research Projects Agency's (DARPA) SyNAPSE program (2014), HICANN as part of the University of Heidelberg's FACETS/BrainScaleS project (2010), and University of Manchester's neuromorphic chip as part of the SpiNNaker project (2014); the latter two are under the flagship of the European Commission's Human Brain Project [29]. All these approaches represent compromises between a set of desirable objectives [18], and their full potential has yet to be realised. Energy-efficiency, integration density, flexibility and configurability, analogue versus digital algorithms, hardware versus software, are all factors that find different balances in the systems listed above.

\section{Emerging areas in neuromorphic computing}

To fully harness the computing power of neuromorphic systems, it is necessary to build artificial synapses and neurons able to mimic the complex dynamics of the biological counterparts. Traditional CMOS systems carry out synaptic plasticity and more in general brain-inspired computing approaches inefficiently since they are inherently volatile, binary and poorly scalable [30]. This is not surprising given that transistors were not created or optimised for this purpose. Devices based on new physical principles are required to replicate biological synapses and neurons in a novel, bio-realistic computing paradigm consistent with the ultra-high density of connections in the human cortex and the complexity of processes such as STDP.

Recently there has been growing interest in memristors and phase-change memories due to their intrinsic similarities to biological synapses. Although these devices do not feature in current large-scale projects, they may well play a major role in future neuromorphic systems, as we will discuss in the next section.

\section{Memristive computing}

In addition to acquiring sensory information from the external environment and producing an appropriate response, the brain is constantly learning from the sensory experiences and from the consequences of its actions. These processes can cause lasting changes in the synaptic weights that make possible to retain the information learned. To artificially mimic the synaptic connections and implement the learning process, it is necessary to build devices able to change their physical properties according to the stimuli received during the learning phase and to retain the changes over time.

In 1971 Leon Chua deduced from symmetry arguments that besides the resistor, the capacitor and the inductor, there should be a fourth fundamental passive circuit element, which he called a memristor, the abbreviation of memory resistor [31]. Chua identified six different mathematical relations connecting pairs of the four fundamental 
circuit variables: electric current $i$, voltage $v$, charge $q$ and magnetic flux $\phi$ (Fig. 3a). The property of the memristor is the memristance $(\mathrm{M})$ and provides a functional relation between charge and flux, $\mathrm{d} \phi=M \mathrm{~d} q$ [32]. In a circuit, if the charge flows in one direction through a component possessing memristance, the resistance of that component will increase, and if the direction of the charge is inverted, the resistance will decrease. If the flow of charge is stopped by turning off the applied voltage, the component retains the memory of the last resistance. The description of a memristor functioning principle and its signature is a Lissajous figure, a pinched hysteresis loop in the voltage-current plane when any bipolar periodic voltage is applied to the device, for which the current is zero when the voltage is zero. The memristor is considered a fundamental element due to the inability to duplicate its properties using other passive elements. However, in practice a real memristor may also possess small amount of other properties, such has capacitance.

After the seminal work of Chua, several publications [33-37] have reported memristive phenomena without naming it as such and without link to Chua's theory. In 2008, Sturkov et al. were the first to present a physical model of a two-terminal electrical device that, under certain conditions, behaves like a perfect memristor [32]. The researchers created a circuit model that implemented the mathematical equations predicted by Chua for the memristor [38], with the exception that Sturkov's memristor had an upper bound to the resistance, meaning that it behaves as a memristive device for large bias or long times (Fig. 3b) [39]. This work explained that the hysteresis that was being observed in the current-voltage (I-V) curves of a wide variety of materials was the result of memristance, or more in general memristive behavior.

Since the first demonstration of memristors, extensive research has been done in the field. The non-volatile multi-state programmability, which ensures that once the programming voltage is removed the desired state is retained without any extra energy consumption, combined with their simple and scalable design, makes memristors promising candidates for implementing artificial synapses and neurons in large-scale neuro-inspired hardware.

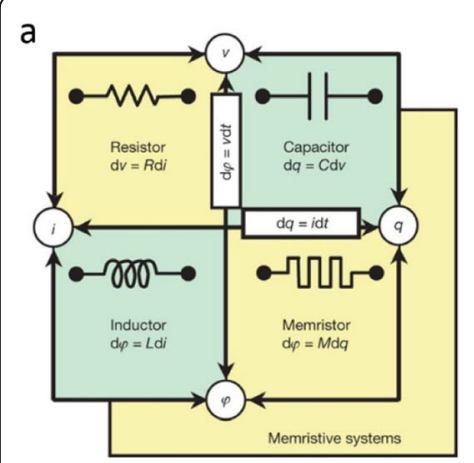

b
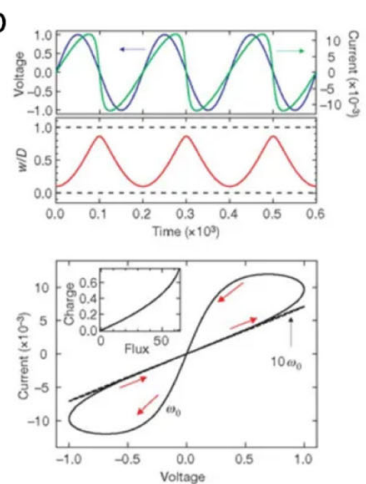

C
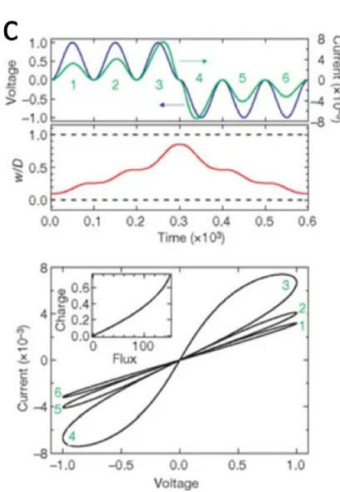

Fig. 3 a Schematic of the fundamental circuit elements: resistor, capacitor, inductor and memristor. b, c The plots show the applied voltage (blue) and resulting current (green) as a function of time $t$ for a typical memristor. In $\mathbf{b}$ the applied voltage is $v_{0} \sin \left(\omega_{0} t\right)$ and the resistance ratio is $R_{\text {off }} / R_{o n}=160$. In $\mathbf{c}$ the applied voltage is $\pm v_{0} \sin ^{2}\left(\omega_{0} t\right)$ and $R_{\text {off }} / R_{\text {on }}=380$. The numbers $1-6$ on the top plot in $\mathbf{c}$ indicate the successive waves in the applied voltage and label the corresponding loops in the $i-v$ curves. The insets in the $i-v$ plots in $\mathbf{b}$ and $\mathbf{c}$ show that in these cases the charge is a single-valued function of the flux, as it must be in a memristor. Reproduced from ref. [32] with permission 


\section{CMOS-memristive neuromorphic hardware}

The use of memristors as artificial synapses for neuromorphic hardware has followed relatively naturally. The synapse is the functional contact point between two neural cells, which guarantees the passage of the excitement from a neuron to the other in only one direction (Fig. 2). An artificial plastic synapse requires three parts to function: a unit to store the synaptic weight, a system to update this weight as a function of the network activity, and finally a circuit to transfer the information between two neurons [40]. Memristors, changing their conductance as a function of the history of voltage differences across the device and retaining the memory of the last resistance, replicate several aspects of synaptic plasticity, like weight evolution, and combine it with weight storage and weight effect.

In 2010 Jo et al. experimentally implemented synaptic functions using nanoscale silicon-based memristors in a two-terminal synapse with crossbar configuration [13]. They were able to achieve STDP in a hybrid synapse/neuron circuit composed of complementary metal-oxide semiconductor (CMOS) neurons and nanoscale memristor synapses, demonstrating the usefulness of memristors in neuromorphic hardware components (Fig. 4).

Since the pioneering work of Jo et al. in 2010 [13], the usage of memristors combined with CMOS technology for implementing artificial synapses in a compact space has seen a large deal of interest [41-44]. More recent experiments focused on the realisation of much larger and more complex memristive neuromorphic networks [45]. Among the others, Eryilmaz et al. in 2014 produced phase-change memristive crossbar arrays devices [46], requiring a transistor at each cross-point. One year later, Prezioso et al. [47] used memristors integrated into a dense, transistor-free crossbar circuit to experimentally demonstrated a single-layer perceptron, with device variability sufficiently low to allow the simple integrated neural network to be taught in situ using a coarse-grain variety of the delta rule algorithm to perform image classification.

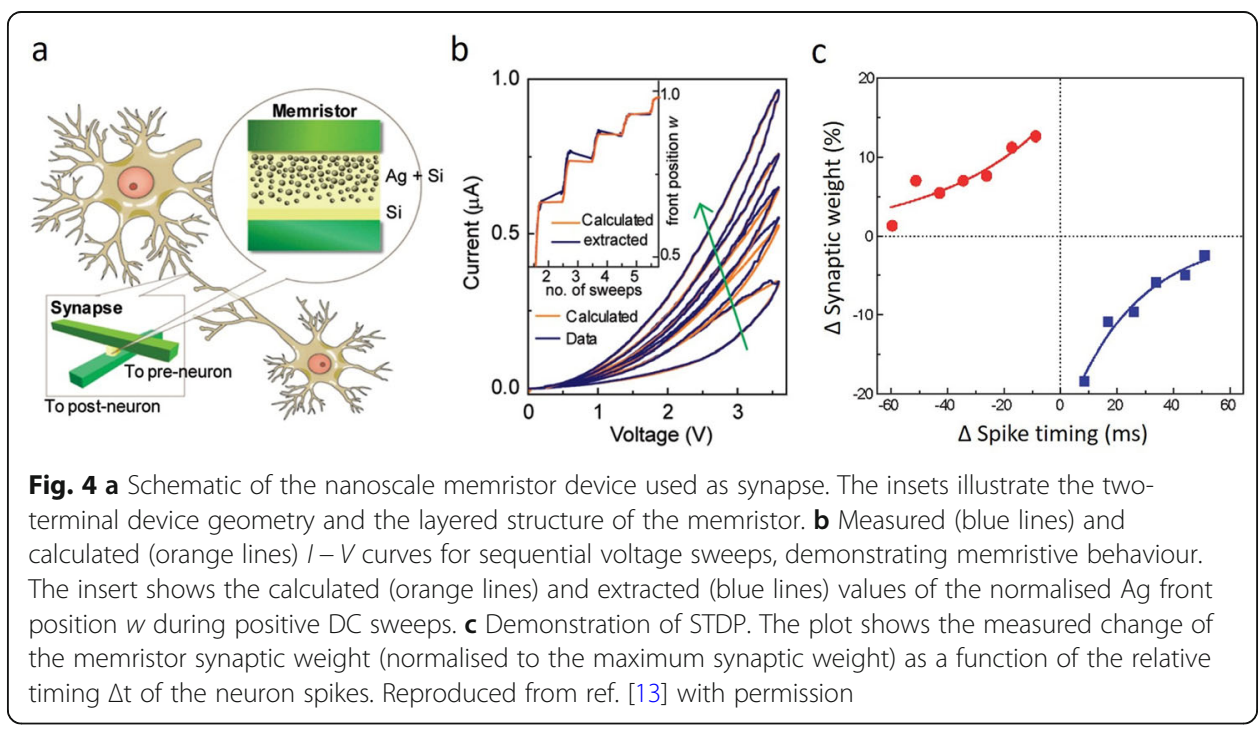


Fully-memristive neuromorphic hardware

In the works reviewed in the previous section, the signal processing functions were implemented either by CMOS circuits (with about 10 transistors or more) or in software running on processors to simulate neurons, which limits further improvements on scalability, bio-realistic dynamics, direct interaction with the artificial synapses, unsupervised training and energy efficiency. All the CMOS limitations raise the interest in developing physical systems able to emulate biological neural systems more directly, to improve efficiency, functionality and scale.

In 2012 Pickett et al. [48] demonstrated a neuristor built using two nanoscale Mott memristors exhibiting all-or-nothing neural functions using materials and structures suitable to extremely high-density integration without silicon transistors. Lately, other artificial neurons based on Mott memristors [49, 50], phase-change memristors [51], redox memristors and chalcogenide threshold switches have been reported with temporal synaptic integration [52].

More recently, Wang et al. [30] achieved the first demonstration of a discrete scalable electronic device that carries out the LIF signal processing and unsupervised learning with memristive synapses (Fig. 5). The artificial neuron was based on a diffusive memristor that relies on the migration of silver in a dielectric host, and it has been used to implement convolution layers, rectified linear units and fully connected layers of a functioning neural network. Pattern classification was achieved using unsupervised synaptic weight update in the fully memristive neural networks.

Memristors have been successfully employed in basic neuromorphic hardware architectures, however creating brain-like hardware-based systems with more advanced functionalities will require an improved understanding of the functionalities of neurobiological systems, the physics of memristors and overcoming several manufacturability challenges [53].

\section{Neuromorphic photonics}

As we have seen in the previous sections, the development of the electronics field has been impressive, however this technology is reaching its physical limitations. Some examples are electronic microprocessor with clock rates unable to exceed about four $\mathrm{GHz}$ before hitting thermal-dissipation limits and parallel architectures limited to even slower timescales. There are also intrinsic bandwidth limitations and the power density of microelectronic chips no longer stays constant as they get denser, that is, smaller transistors do not consume less power [54]. Current electronic implementations of new information processing architectures, such as neuromorphic architectures are still far from competing with biological neural systems in terms of real-time information-processing capabilities, packing density and energy efficiency. The time is fast approaching for a shift towards dramatically different technology [55].

A solution to the heat generation and bandwidth limitations is represented by photonic technology, which can provide energy-efficient passive components, low heat generation, low crosstalk and parallel processing. But photonics can go beyond being just a convenient way of transferring data. Many physical processes employed in photonic devices can be described within the framework of nonlinear dynamics, and for this reason have a strong analogy with several biological processing models. 


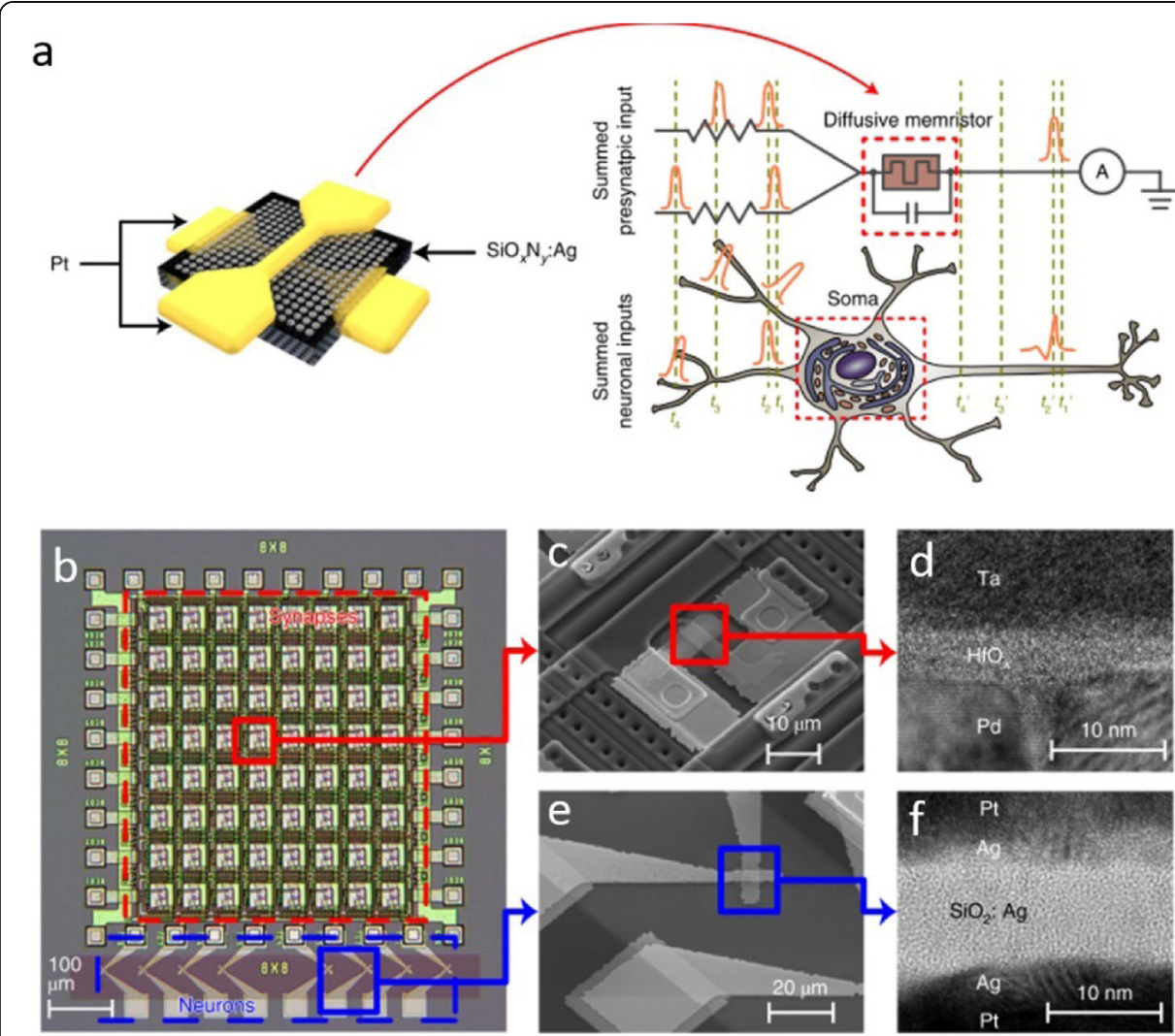

Fig. 5 a Schematic of the crosspoint diffusive memristor consisting of a SiOx Ny:Ag layer between two Pt electrodes. The artificial neuron receives software-summed presynaptic inputs via a pulsed voltage source and an equivalent synaptic resistor. Both the artificial and biological neurons integrate input stimuli (here represented in orange) and fire when the threshold condition is reached. $\mathbf{b}$ Optical micrograph of the integrated memristive neural network, consisting of an $8 \times 8$ one-transistorone-resistor memristive synapse crossbar interfacing with eight diffusive memristor artificial neurons. $\mathbf{c}$ Scanning electron micrograph of a single synaptic cell. $\mathbf{d}$ Cross-sectional transmission electron microscopy image of the integrated $\mathrm{Pd} / \mathrm{HfOx} / \mathrm{Ta}$ drift memristor prepared by focused-ion-beam cutting. e Scanning electron micrograph of a single diffusive memristor junction. $\mathbf{f}$ High-resolution transmission electron micrograph of the cross-section of the Pt/Ag/SiOx:Ag/Ag/Pt diffusive memristor showing amorphous background SiOx with nanocrystalline thin Ag layer. Reproduced from ref. [30] with permission

The application of optical networking principles to the neuromorphic domain creates the field of neuromorphic photonics, which combines the advantages of photonics and neuromorphic architectures to build systems with high efficiency, high interconnectivity and high information density, and paves the way to ultrafast, low cost and complex signal processing without consuming impractical amounts of power (Fig. 6).

Despite all the arguments in favour of the application of photonics to the neuromorphic domain, it is not yet clear if neuromorphic photonics can compete with its electronic counterpart. Until now, the complex applications of photonic neuromorphic hardware have remained largely unexplored due to difficulties in the optical implementation of memory and logic and the absence of a robust photonic manufacturing and integration industry. Nevertheless, neuromorphic photonic architectures hold a lot of potential and the technological developments achieved so far are promising starting points for future developments. 


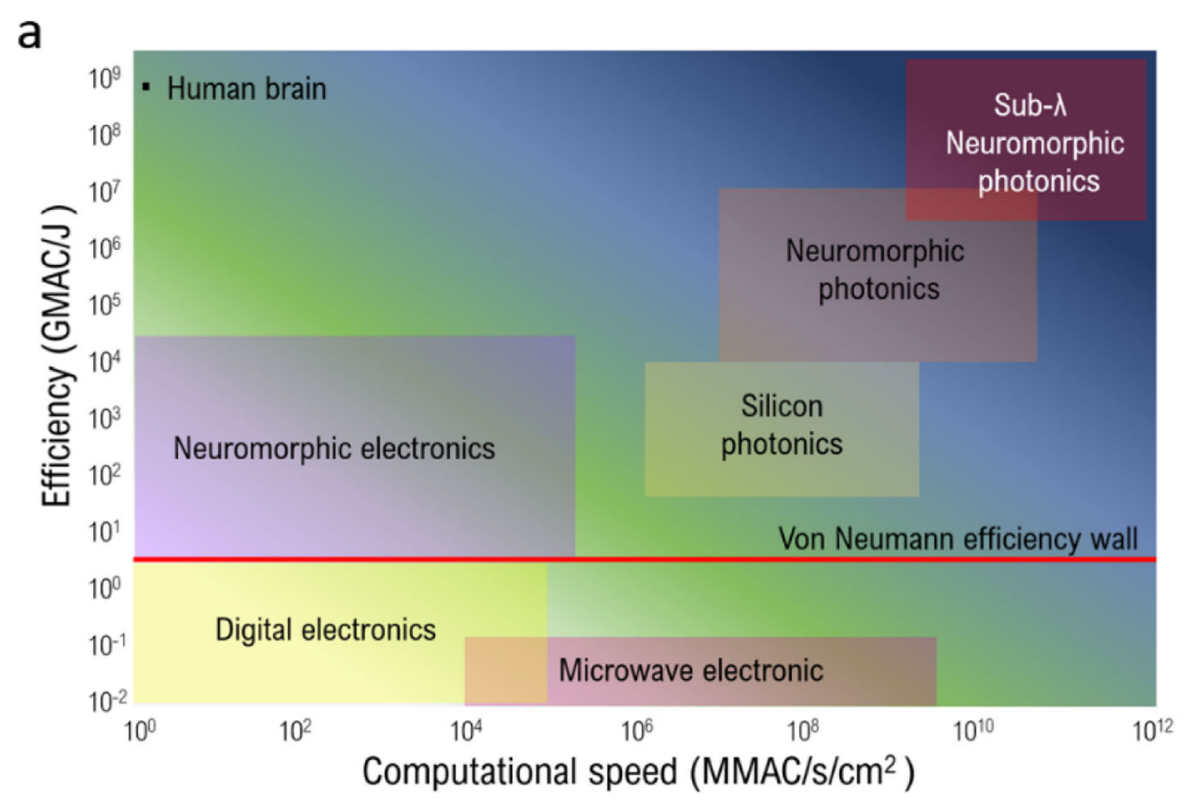

b
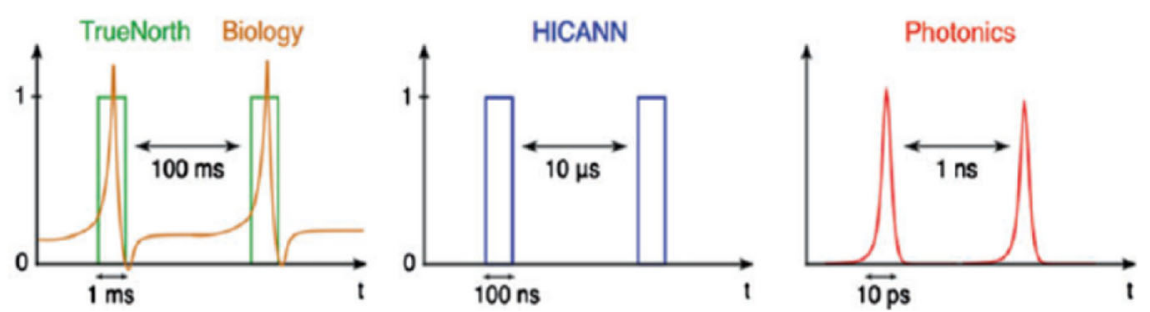

Fig. 6 a Qualitative comparison of computational speed and efficiency for different technologies: human brain, digital electronics, microwave electronics, neuromorphic electronics, silicon photonics, neuromorphic photonic and super-resolved neuromorphic photonics. b Timescale comparison between TrueNorth, biological neuron, HICANN and photonics spike processing. Reproduced from ref. [56] with permission

\section{Early works}

The investigation of photonics for neuro-inspired information processing has taken place alongside the development of electronic neuromorphic architectures. The first optical neural network ever made is attributed to Psaltis and Farhat in 1985 [57], who showed that pattern retrieval can be achieved with a lenslet array interconnection network. Since then, several works used free space optics and fibre components to build optical neural networks, but undeveloped fibre-optic and photonic technologies and limited scalability strategies relegated these systems to laboratory demonstrations [58-60].

Photonic technology and neuroscience have matured enormously since then, allowing photonic neuromorphic research to progress. The first demonstration of photonic spike processing was achieved only in 2009 by Rosenbluth [61]. Spike processors, like gates in digital computers, act as baseline units of larger interconnected networks able to perform more complex computations. Rosenbluth's photonic integrate-and-fire systems, based on optical spike processing devices, operated on picosecond width pulses and had an integration time constant in the order of $100 \mathrm{ps}$, about eight orders of magnitude faster than biological neurons. 
A bloom in research related to various aspects of photonic spike processing followed Rosenbluth's first demonstration [62-64]. Many of these proposals for spiking "photonic neurons" or "laser neurons" or "optical neurons" have been extensively reviewed [54, 65, 66].

\section{Evolution of the field towards photonic plasticity}

One of the most appealing features of neural systems is their plasticity, i.e. the natural ability to learn and dynamically adapt to an unpredictable and changing environment. Therefore, a vital stage towards the development of truly bio-realistic photonic neuromorphic hardware is the photonic implementation of plasticity in artificial synapses and neurons.

Photonic synapses designs have been proposed to broaden the bandwidth and mitigate the interconnect issues of electronic synapses. Several implementations of STDP circuits [67], and photonic synapses [68, 69], demonstrated the benefits of large bandwidth and fulloptical interconnections [55]. However, the synapses realised in these works tend to be either difficult to integrate and speed-limited, or still rely on electrical excitation signals.

In 2017, Cheng et al. [70] demonstrate a fully integrated all-photonic synapse based on phase change materials (PCMs), implemented via a photonic integrated-circuit approach (Fig. 7). Their device resembles the neural synapse at the physical level and can achieve synaptic plasticity compatible with the STDP rule. A following work proposed an allphotonic integrate-and-fire spiking neuron [71], with the potential to be integrated in a spiking neural network. This neuron does not implement any form of plasticity, nevertheless is a plausible replication of biological functionalities. More recently, Feldman et al. demonstrated an all-optical neurosynaptic system capable of supervised and unsupervised learning implementing a simplified STDP rule through ring resonators and PCM units

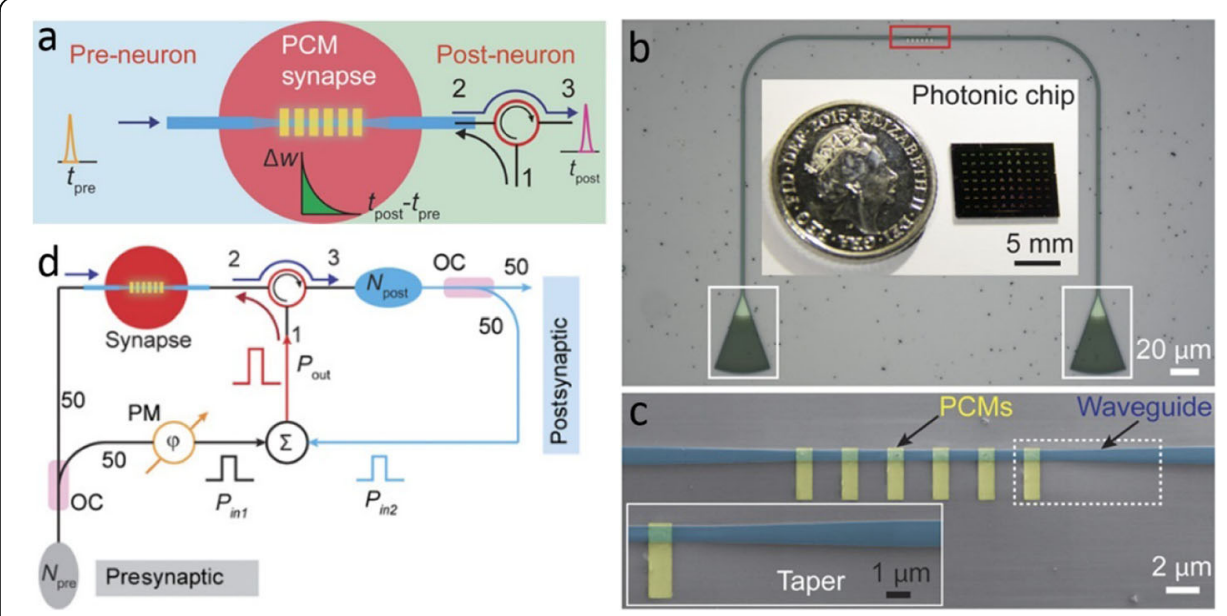

Fig. 7 a Schematic of the photonic synapse. The synapse optically connects the presynaptic (pre-neuron) and the postsynaptic (post-neuron) signals and it consists in a tapered waveguide (blue) with discrete PCM islands on top (yellow). The red open circle is a circulator with port 1 receiving the weighting in input, and port 2 and port 3 connecting the synapse and the post-neuron. $\mathbf{b}$ Optical microscope image of a device with the active region (red box) as the photonic synapse. Inset: A typical photonic chip containing 70 photonic synapses has a dimension smaller than a 5-pence coin. c Scanning electron microscope image of the active region of the photonic synapse corresponding to the red box in (b) with six GST units ( $1 \mu \mathrm{m} \times$ $3 \mu \mathrm{m}$, yellow, false-coloured) on top of the waveguide (blue, false-coloured). Inset: The zoomed-in tapered structure of the waveguide highlighted by the white dashed box. $\mathbf{d}$ Schematic of the all-optical method using a photonic synapse to achieve the STDP plasticity. Reproduced from ref. [70] with permission 
(Fig. 8) [72]. These works show that the direction that neuromorphic photonic is undertaking is towards the implementation of all-photonic verisimilar neural networks. Following the logic underneath the development of the neuromorphic electronics field, volatile- and non-volatile photonic memories will be essential for the photonic implementation of plasticity and to harvest the full potential of neuromorphic photonics.

Other notable works in the photonic neuromorphic domain include applications such as deep neural networks [73], and recurrent neural networks [74]. These works purely aim to implement different computational models with optics, therefore are complementary to the processing framework discussed in this paper.

Even if a significant effort has already been done towards the emulation of learning and thinking processes of the human brain, the development of the next generation of neuromorphic photonic hardware with a wider range of biomimetic functionalities presents several technical and scientific challenges. In what follows we will discuss the role of photonic analogue of memristors, which we believe will have a fundamental role in the development of photonic neuromorphic hardware, as their electronic counterpart had in the development of neuromorphic electronics.

\section{Photonic memristors}

Memristors have been largely used to build artificial synapses since their history-dependent conductivity modulation is intrinsically similar to several aspects of synaptic plasticity, such

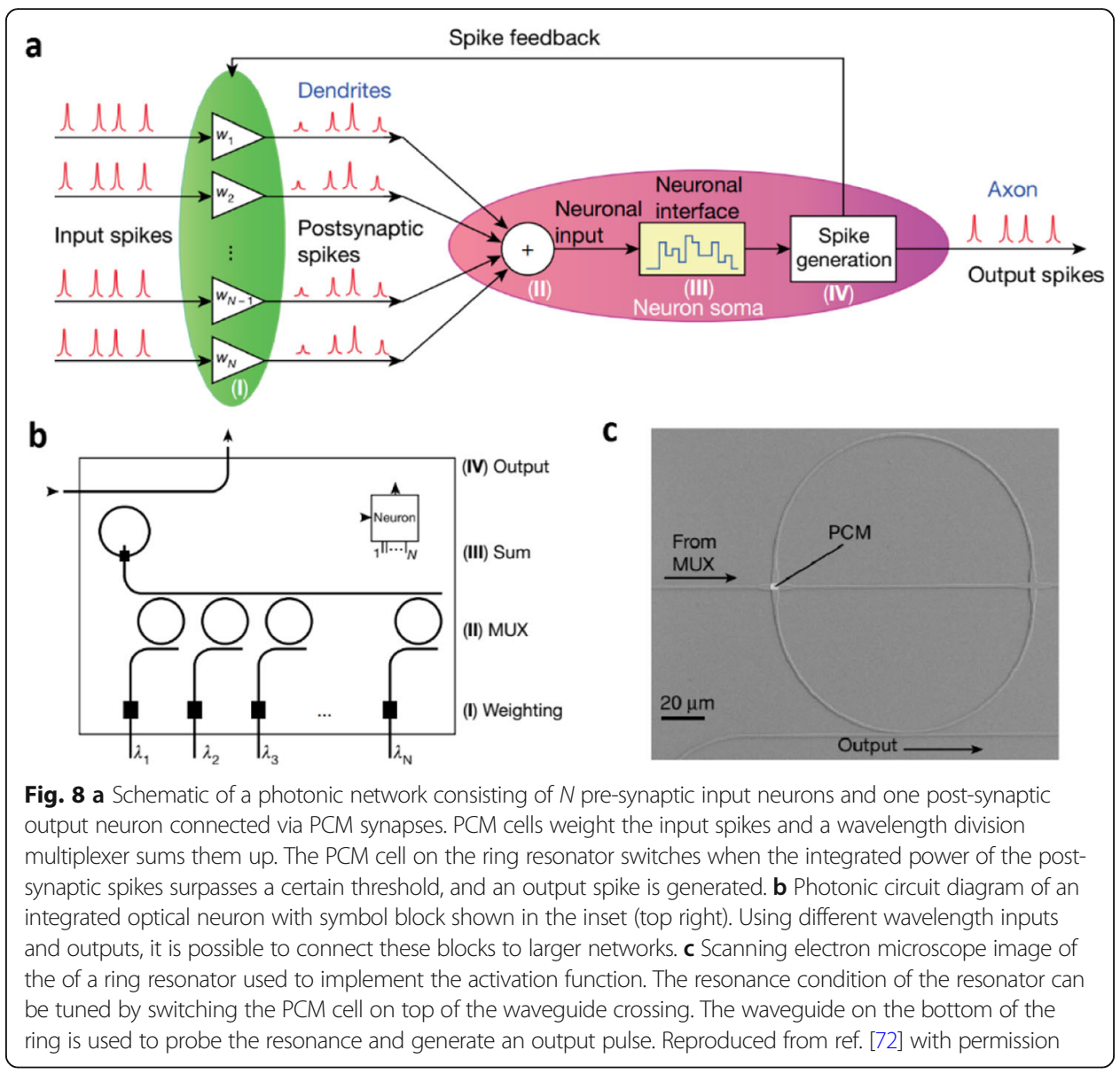


as weight update and weight storage. At first, memristive components were combined with CMOS technology to build synapses [13], and later on, complete neural networks [47]. More recently, fully memerisitive neuristors [48], and neural networks [30], have been realised to avoid the use of transistors and improve scalability, stackability and energy efficiency.

Similarly, in the field of neuromorphic photonics we are witnessing increasing efforts to replicate synapses and neurons, and the use of photonic memory elements is playing a crucial role [70, 72]. To achieve truly biomimetic neuromorphic photonic hardware, we believe that the photonic equivalent of memristors is needed. In what follows, we discuss the possibility to conceive photonic memrisistive systems.

\section{State of the art}

The fingerprint of electronic memristors are the hysteresis $I-V$ loops, the demonstration that these systems possess a memory of the last charge that passed through them. For the nonvolatile memristors the memory is retained even in absence of current (Fig. 9a), but in the volatile case the memory is lost when the charge has been removed (Fig. 9b). Ideally, in a photonic memristor the transmittance changes in relation to the electromagnetic field incident on the device. Therefore, photonic memristors will exhibit hysteretic transmission-incident field power (T-P) loops similarly to the hysteretic $I-V$ loops of electronic memristor. This effect can be transient and disappear when the incident electromagnetic field is interrupted or persistent, even in absence of incident magnetic field. The first case will be referred to as memristive-like behaviour and the second as permanent memristive behaviour.

Some attempts to realise photonic memrisistive systems have already been made by Emboras et al. [76] with the demonstration of a nanoscale plasmonic memristor characterised by electrical writing and optical readout functionalities (Fig. 9c and d). In this case the memristive behavior was attributed to the optical bistability (OB) of the fundamental plasmonic mode resulting from the voltage induced annihilation of the nanoscale metal filament. The advent of metamaterials, media with artificially design electromagnetic properties, unlocked further possibilities. Metamaterials can be designed to display nonlinearity when interacting with electromagnetic waves, and for some designs, they can achieve photonic memristive-like behaviours. Wu et al. [77] observed photonic memristive-like behaviour in dielectric metamaterials consisting of $\mathrm{CaTiO}_{3}-\mathrm{ZrO}_{2}$ ceramic dielectric cubes in the microwave wavelength region (Fig. 9e and f), and measured hysteretic T-P loops in these systems. The effect was attributed to the decreasing permittivity of the dielectric cubes caused by the increasing temperature generated by the interaction with the electromagnetic waves [77].

The first critical step towards the practical realisation all-photonic memristive hardware with operative wavelength in the optical domain is the material selection for the single units where memory and signal processing take place.

\section{Laser-patterned graphene photonic memristors}

Third order optical nonlinearities can potentially lead to permanent or memristivelike behaviour depending on the amount of time the new state can be kept for. $\mathrm{OB}$ is one remarkable feature of nonlinear optical effects $[78,79]$, where a nonlinear optical system exhibits two distinguished stable excited states for a single input intensity. Relying on optical non-linearity, OB materials can exhibit memristive-like and, under certain circumstances, memristive behaviour. 
a

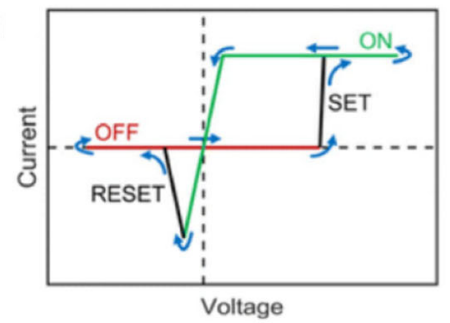

C

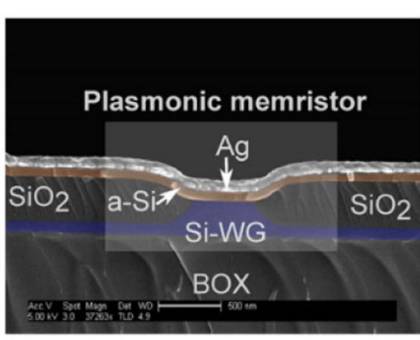

e

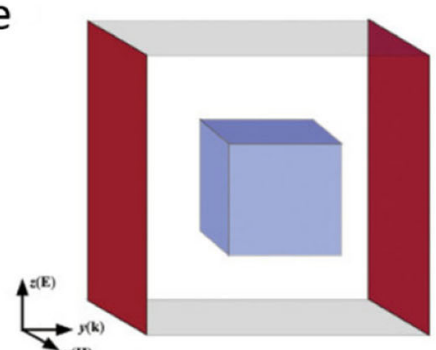

b

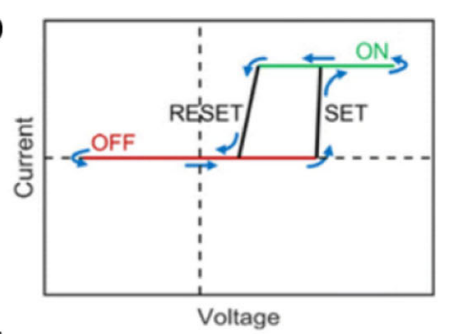

d

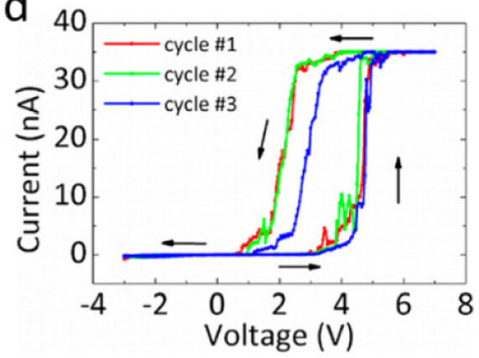

$f$

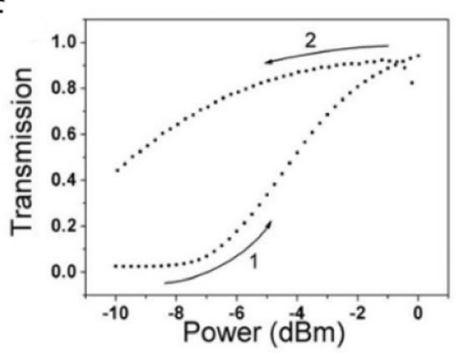

Fig. 9 Typical I-V curve of non-volatile (a) and volatile (b) memristive switches. Reproduced from ref. [75] with permission. c Plasmonic memristor. Scanning electron microscope image of the cross section of the optically readable memristive RRAM. d Measured I-V curves for subsequent cycles (indicated by the different colours) of the plasmonic memristor device that consist of Ag/a-Si/pSi layer fabricated on SOI wafer. Reproduced from ref. [76] with permission. Microwave memristive-like behaviour in dielectric memtamaterials. e Schematic diagram of the unit cell of one dielectric cube. The incident electromagnetic wave propagates along the $y$ axis, and the electric field and magnetic field propagate along $z$ and $x$ directions, respectively. f T-P plot of the metamaterials for a scan rate of $1 \mathrm{dBm} / \mathrm{s}$ at $11.68 \mathrm{GHz}$. The T-P plot of the resonance peak as the output power of the vector network analyser increased from $-10 \mathrm{dBm}$ to a maximum $\left(P_{\max }\right)$ of $0 \mathrm{dBm}$ at a scan rate of $1 \mathrm{dBm} / \mathrm{s}$. The power was subsequently decreased from $P_{\max }$ to -10 $\mathrm{dBm}$ at the same rate. Reproduced from ref. [77] with permission

Since the last century, graphene has been considered an outstanding electronic and optical material due to its flexibility, robustness, optical transparency, extremely large electron mobility and tuneable nonlinear optical conductivity [80-83]. Moreover, graphene's intense third-order optical nonlinearity has been quantified to be of order $10^{-11}$ $\mathrm{m}^{2} / \mathrm{W}[84]$, and its extraordinary plasmonic field enhancements have attracted significant interest in the plasmonic community [80]. Giant optical nonlinearity and OB has also been investigated in graphene $[85,86]$, graphene-silicon waveguide resonator, graphenesilicon photonic crystal cavities and functionalised graphitic carbon nitride [87].

Graphene Oxide (GO) is a single monomolecular layer of graphite with several oxygen-containing groups, a strongly hydrophilic intermediate product to chemically form graphene. It has attracted attention among cross-discipline researchers due to its tuneable electrical and optical properties dynamically controllable by the reduction of oxygen-containing groups manipulating through either chemical [88], or physical reduction methods such as laser exposure $[89,90]$. Tuning of the nonlinear absorption 
response, switch of the nonlinear refractive index and giant Kerr nonlinear response are observed during the transition from GO to reduced GO (rGO) [89]. Moreover, the oxygen-containing groups enable an easy functionalization of $\mathrm{GO}$ and $\mathrm{rGO}$ with a variety of functional materials such as organic and inorganic molecules.

As we can see in Fig. 10, GO and rGO third-order optical nonlinearity generates OB that can be harnessed to achieve optical memristive-like behaviour. The linear and nonlinear refractive index of rGO can be finely tuned by controlling the oxidation level of the materials with femtosecond laser, resulting in modular T-P loops (Fig. 10c and d) [89]. Controlling the photoreduction process, the memristive properties of rGO can be finely tuned.

To achieve non-volatile optical memristive behaviour, a solution can be considering innovative photosensitive materials resulting from the combination of graphene and GO with PCMs [91].

\section{Alternative materials}

Using $\mathrm{OB}$ materials, it is possible to achieve Page 22:, but it can be hard to realise permanent photonic memristors, as required by a variety of applications. Different mechanism can lead to non-volatile memory and to memristive behaviour such as phase transition effect, valency change effect, electrochemical metallization and phase change effect [75]. In what follows, we propose different materials suitable to achieve

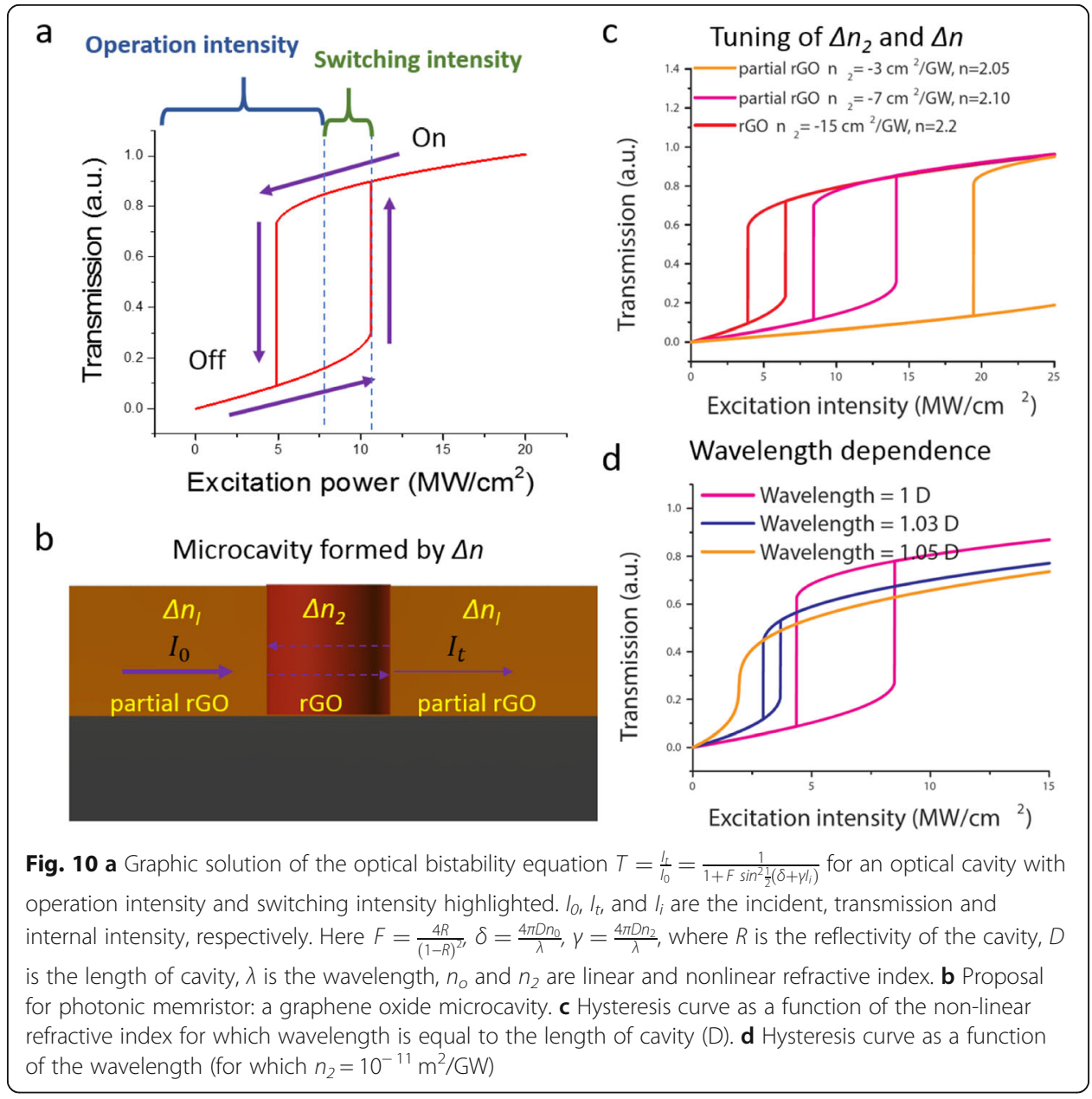


photonic memristive and memristive-like behaviour focusing on bistable and phase change materials. We will quantitatively compare nonlinear refractive indexes, and optical properties for the different phases.

\section{Optical phase change materials}

Optical phase change materials (OPCM) are a class of materials that exhibit large changes in their optical properties in response to an external stimulus [92]. They are excellent candidates for achieving true nonvolatility and memristive behaviour since they show nonvolatile and extraordinary large changes in the electronic and optical properties during structural or electronic phase transitions. The transitions can be driven by either temperature changes, electrical or optical pulses and the cycle between these states is reversible and reproducible under appropriate thermal, electrical, or optical stimulation [93].

OPCMs devices have been shown to emulate spiking neurons and plastic synapses. For example, synaptic weight depression and potentiation can be achieved by partial amorphization and progressive crystallization of OPCM components under optical excitations [71], or the STDP rule can be implemented combining multiple optical pulses with different amplitudes, widths and time intervals [94]. In addition, phase-change photonic networks can perform fundamental arithmetic operations by all-optical methods [95]. To date, the most extensively studied OPCMs have been vanadium dioxide $\left(\mathrm{VO}_{2}\right)$ and $\mathrm{Ge}_{2} \mathrm{Sb}_{2} \mathrm{Te}_{5}$ (GST), and in what follows we discuss their application in photonic memristors and memristive-like devices.

\section{GST}

$\mathrm{Ge}_{2} \mathrm{Sb}_{2} \mathrm{Te}_{5}$ (GST) is a chalcogen-based OPCM that undergoes a rapid and reversible amorphous-crystalline transition from an amorphous state optically transmissive and electrically resistive, to a crystalline state optically opaque and electrically conductive [92]. Once the phase transition is concluded, the amorphous and the crystalline states are both non-volatile, making GST a well suited material for non-volatile optical memories and potentially for photonic memristors.

Combining GST and nanophotonics, it has been possible to demonstrate multibit non-volatile all-optical memories. By incorporating GST in a photonic circuit, Ríos et al. achieved eight memory levels in a single device, using optical pulses for write, read, erase and switch memory levels [96]. The pulse-width modulation scheme used in this work enables the direct writing of a specific memory level from any starting state using the same pulse-width modulation pulse sequence. Since then, GST has been wildly studied and multilevel controlled switching [97], and a non-volatile quasicontinuously reprogrammable platforms have been achieved [98].

GST possess also nonlinear properties that can be exploited for the realisation of photonic memresistive-like behaviour in case that volatility is required. Liu et al. [99] provided a theoretical explanation and a quantitative determination of optical nonlinearities of crystalline GST. Their analysis indicates nonlinear saturable absorption mainly due to band-filling effect at low laser intensity $\left(<0.17 \mathrm{GW} / \mathrm{m}^{2}\right)$, and nonlinear reverse-saturable absorption due to thermal-induced absorption increment at high laser intensity $\left(>0.17 \mathrm{GW} / \mathrm{m}^{2}\right)$. 
$\mathrm{VO}_{2}$

$\mathrm{VO}_{2}$ is a metal oxide OPCMs that exhibits a symmetry-lowering structural phase transition due to a strongly correlated electron system, leading to a large heat of transition and demonstrating in this way a great potential as OPCM [100]. More specifically, $\mathrm{VO}_{2}$ undergoes both insulator-to-metal and crystalline-crystalline transitions, from a monoclinic state (optically transmissive, electrically resistive) to a rutile state (optically opaque, electrically conductive). This transition can be accessed thermally, electrically, or with ultrafast optical excitations [75, 100]. The $\mathrm{VO}_{2}$ monoclinic state exists at room temperature and ambient pressure, making $\mathrm{VO}_{2}$ volatile and potentially well suited for applications such as modulation [92], or photonic memristive-like devices.

In addition to the transport properties discussed above, $\mathrm{VO}_{2}$ shows a variety of nonlinear optical properties, especially in combination with other materials. For example, nanocrystalline $\mathrm{VO}_{2} / \mathrm{SiO}_{2}$ composite films exhibit a negative nonlinear refractive index and saturable absorption behavior, when laser pulses induce the metal-semiconductor phase transition. The sign of the nonlinear refractive index flips in the phase transition [101], and the value of the non-linear absorption coefficient can be modulated by the pulse duration of the incident light [102]. These properties make $\mathrm{VO}_{2}$ and $\mathrm{VO}_{2}$-composite materials attractive for prospective memristive-like and optoelectronic applications such as bistable ultrafast optical switchers and memory.

\section{Other chalcogenide glasses}

Chalcogenide glasses are an entire family of materials that can switch between glassy and crystalline phase. A pronounced change in the optical properties accompanies the electrical resistance contrast during the fast and reversible phase changes of chalcogenide materials. Refractive index, extinction coefficient and absorbance change by several tens of percent between glassy and crystalline phase, due to a change from covalent to metavalent bonding. The sharp optical contrast leaded to chalcogenide OPCM-based rewriteable optical data storage devices in the 1990s [103]. Moreover, chalcogenide glasses display high linear and non-linear refractive indexes and photoinduced phenomena, such as photodarkening and photocrystallization, typically several orders of magnitude higher than in fused silica [104].

The progress in nanophotonic technologies combined to the non-linear optical properties and the phase change nature of chalcogenide glasses open up the possibility to explore the use of chalcogenide-based materials, such as the recently discovered $\mathrm{Sc}_{0.2} \mathrm{Sb}_{2} \mathrm{Te}_{3}$ [94], for the realisation of on-chip photonic phase-change memories, highspeed and high-capacity optical communication networks and photonic memristive neuromorphic devices.

\section{Antimony telluride}

Antimony telluride $\left(\mathrm{Sb}_{2} \mathrm{Te}_{3}\right)$ and other lead-based phase OPCMs are extensively applied in optical data storage [105, 106], photo-lithography [107, 108], holography [109], and topological photonics [110]. All these applications depend on the response of the material to the applied electromagnetic field, the temperature or the interaction between material and laser pulses [111].

The crystalline $\mathrm{Sb}_{2} \mathrm{Te}_{3}$ films used for core-cladding structures and mask layers exhibit a temperature-dependent transmittance, a giant optical nonlinear absorption and 
refraction under low laser intensity [112], due to saturation of direct bandgap transition and thermal effect, respectively [111]. The optical non-linearity and the dramatic changes in the optical properties with the phase and the film thickness indicate that the $\mathrm{Sb}_{2} \mathrm{Te}_{3}$ and more in general Sb-based materials are promising candidates for both volatile and non-volatile photonic memristive devices.

\section{Silicon}

Silicon has been widely used in photonics due to its potential to high density, power efficiency, and its compatibility with CMOS fabrication technologies. Despite the potential for low-cost mass production and high compatibility with CMOS industry, the use of silicon, the material of choice for very large-scale integration circuits in the conventional von Neumann computing, has barely been explored for the realisation photonic neuromorphic hardware [74].

The potential of silicon as photonic neuromorphic material lies in the variety of nonlinear effects that can be used to detect and process optical signals, or generation of photons for lasing and amplification [113]. Third-order nonlinearities are especially important as they entail a wide variety of phenomena such as self-phase modulation, intensity-dependent refractive index change, two photon absorption and can therefore lead to the possibility of photonic memristive-like behaviour. The silicon Kerr coefficient peaks around $1.85 \mu \mathrm{m}$ beyond half the bandgap energy, and is therefore expected to shows very favourable Kerr nonlinearities with a large figure of merit in the infrared wavelength region [113].

To exploit silicon nonlinear effects, light must be channel into waveguides [113], or trapped in photonic crystals nanocavities [114], where the nonlinear processes can take place. Additionally, silicon can be combined with OPCMs for the realisation of thermooptic, electro-optic, and all-optical Si/OPCM devices [92], and the achievement of the photonic memristive effect.

To evaluate the materials discussed above, in Table 1 we compare their refractive index, nonlinear refractive index and extinction coefficient at two different wavelengths. The nonlinear optical materials in Table 1a are volatile and are suitable for realising

Table 1 Comparison of refractive index $(n)$, nonlinear refractive index $\left(n^{2}\right)$ and extinction coefficient (k) at two different wavelengths for optical nonlinear materials (a) and OPCM (b)

\begin{tabular}{|c|c|c|c|c|c|}
\hline Material & $\begin{array}{l}n \\
\lambda=600 \mathrm{~nm}\end{array}$ & $\begin{array}{l}n \\
\lambda=1500 \mathrm{~nm}\end{array}$ & $\begin{array}{l}n^{2} \\
\left(m^{2} / W\right)\end{array}$ & $\begin{array}{l}k \\
\lambda=600 \mathrm{~nm}\end{array}$ & $\begin{array}{l}k \\
\lambda=1550 \mathrm{~nm}\end{array}$ \\
\hline \multicolumn{6}{|c|}{ a Nonlinear optical materials } \\
\hline graphene & $2.7[115]$ & $3.1[115]$ & $-1 \cdot 10^{-13}$ at $\lambda=1600 \mathrm{~nm}$ [84] & $1.3[115]$ & $2.2[115]$ \\
\hline $\mathrm{GO}$ & $1.9[116]$ & $2.1[117]$ & $8 \cdot 10^{-13}$ at $\lambda=800 \mathrm{~nm}[118]$ & $0.1[116]$ & $0.3[117]$ \\
\hline reduced GO & $1.8[116]$ & - & $-4.9 \cdot 10^{-13}$ at $\lambda=800 \mathrm{~nm}[119]$ & $0.2[116]$ & - \\
\hline Silicon & $4[115]$ & $3.5[120]$ & $4.3 \cdot 10^{-18}$ at $\lambda=1600 \mathrm{~nm}[121]$ & $0.03[115]$ & $0.01[120]$ \\
\hline \multicolumn{6}{|c|}{ b Phase change materials } \\
\hline GST (amorphous) & $3.2[122]$ & $3.1[122]$ & - & 1 [122] & $0.1[122]$ \\
\hline GST (crystalline) & 3.3 [122] & $4.2[122]$ & - & $1.8[122]$ & $0.8[122]$ \\
\hline $\mathrm{VO}_{2}$ (amorphous) & 3.0 [123] & 3.3 [123] & - & $0.5[123]$ & 0.4 [123] \\
\hline $\mathrm{VO}_{2}$ (crystalline) & $2.2[123]$ & $2.8[123]$ & $7.5 \cdot 10^{-8}$ at $\lambda=800 \mathrm{~nm}[101]$ & $03.9[123]$ & $0.6[123]$ \\
\hline $\mathrm{As}_{2} \mathrm{~S}_{3}$ & $2.7[124]$ & $2.4[124]$ & $3.8 \cdot 10^{-10}$ at $\lambda=1550 \mathrm{~nm}[124]$ & - & - \\
\hline $\mathrm{Sb}_{2} \mathrm{Te}_{3}$ & $1.8[110]$ & $6.0[110]$ & $2.6 \cdot 10^{-9}$ at $\lambda=632.8 \mathrm{~nm}[125]$ & $2.9[110]$ & $0.8[110]$ \\
\hline
\end{tabular}


memristive-like behaviour while the OPCM in Table $1 \mathrm{~b}$ are non-volatile and can be used for permanent photonic memristors. While all the materials listed in Table 1a possess remarkably non-linear optical properties, the switch in sign and the tuneability of the nonlinear refractive of $\mathrm{GO}$ during the reduction process to $\mathrm{rGO}$, make this material the preferential candidate to implement different plasticity mechanisms. Nevertheless, different neuron and plasticity models have different requirements in terms of volatility and signal modulation, and the choice of the material tailored should reflect those requirements.

\section{Applications}

Photonic memristors have the potential to become promising candidates for the next generation photonic neuromorphic computing systems. In what follows we discuss the specific applications they can find.

\section{On-chip processing}

Integrated photonic circuits (IPC) allowe on-chip computing with the high-speed and bandwidth potential of the optical domain, removing the need for electrooptical conversions. Further advantages are information density, scalability, energy efficiency and contained cost. The recent achievements on the field collectively demonstrate the great strides that have been made towards the goal of realising powerful systems-on-a-chip [126].

\section{Neuromorphic photonic hardware}

IPC are systems well suited to implement neuromorphic architectures able to replicate basic neural functional units, namely neurons and synapses. Integrating memristive photonic elements in these circuits will pave the way to a more compact and faithful photonic implementation of a wide class of neuro plasticity mechanisms. Volatile and non-volatile photonic memristive materials can be used to mimic the different plasticity mechanisms that regulate learning and memory, and in this way memristive photonic chips can unlock unprecedent performances.

\section{Photonic in-memory computing}

Photonic memristors can combine integrated optics, data storage and signal processing to enable all-photonic in-memory computing. As we discussed in the previous sections, in von Neumann architectures there is a clear separation between the CPU and the storage unit, and the rate at which data can be transferred represents a fundamental limitation of von Neumann computers, known as the memory wall. In-memory computing is an alternative approach where the computation takes place within the memory, eliminating in this way the energy-intensive and time-consuming data movement of the von Neumann [7]. IPC implementations of in-memory computing have the potential to further transform the computing landscape, by providing ultra-high processing speeds and increased bandwidths that can come from working directly in the optical domain $[7,127]$.

Optically accessible memories are rapidly bridging a gap toward all-photonic chipscale in-memory information processing. The employment of memristive photonic 
elements has the potential to achieve all-optical direct scalar and matrix-vector multiplication exploiting a single-shot write/erase and a drift-free process [127]. In this case, the result of the computation will be the output pulse carrying the information of the light-matter interaction. This memristive in-memory approach will set the stage for the development of entirely photonic in-memory computers.

\section{Parallel processing}

Applications of AI techniques, specifically machine learning and more recently deep learning [128], are transforming several fields ranging from clinical medicine to optical computing. The integration of full-optical neuromorphic architectures with optoelectronic devices will lead to the near-term availability of clinically and industrially relevant applications such as real-time features detection and classification, image processing and optical implementation of computationally intensive tasks such as matrix multiplication with low-power consumption, high-accuracy and ultra-fast processing speed.

Recently, all-optical diffractive deep neural network designs, systems physically formed by multiple layers of diffractive surfaces that work in collaboration to optically perform arbitrary functions, have been investigated in the terahertz [129], and in the optic wavelength region [130]. Incorporating optical nonlinear memory materials like photonic memristors into the diffractive layers will include nonlinear activation functions within the optical networks and will further enhance the inference performance of all-optical diffractive deep neural networks. The change in optical properties induced by the history of the transmitted fields, will open the path to dynamic diffractive processing [131].

\section{Analysis and outlook}

Optically nonlinear, phase change and high refractive index materials are the favoured candidates for the realisation of photonic memristive devices. While the above sections discuss the concept of photonic memristance along with the material candidates, this section examines the challenges in the practical realisation of photonic memristors, reviewing techniques for synthesis, fabrication and assembly of such systems.

Several techniques can be adopted for the fabrication of bulk and thin films of high refractive index materials like $\mathrm{Sb}_{2} \mathrm{Te}_{3}, \mathrm{VO}_{2}$ and chalcogenide glasses such as thermal evaporation [132], flash evaporation [133], sputtering, chemical vapor deposition [134], atomic layer deposition [108], spin coating, and many more. The deposition method to choose depends largely on the material composition that is to be deposited, the desired parameters such as uniformity and thickness, and the final design. For example, for the realisation of single memristive neuromorphic units such as photonic synapses, a conformal thin film coating with thickness precisely tuneable is desirable. On the other hand, IPCs require films with millimetric thickness and high optical quality to minimise waveguide propagation loss.

For materials such silicon and graphene several methods are well known to produce very high-quality thin films with modular thicknesses [113]. However, the fabrication of custom designs with nanometric features for achieving functional neuromorphic units is comparatively challenging and presents opportunity for more research and investigation. 
Achieving GO-based laser patterned photonic memristors with sizes comparable to those of the biological synapses $(20-40 \mathrm{~nm})^{10}$, will require fabrication methods able to go beyond the diffraction limit. Technologies such as super-resolution photoinductioninhibited nanofabrication (SPIN) [135-137], enable rapid prototyping of two-dimensional (2D) and three-dimensional (3D) structures with resolution well below the diffraction limit and can potentially be applied to GO and rGO specimens. A two-beam nanolithography technique based on the control of graphene photoreduction and photooxidation processes by two femtosecond lasers with different wavelength can be used to achieve graphene-based super-resolution patterns. Application of such a method to GO and rGO films should be further explored with the aim to achieve bio-realistic onchip synaptic density and higher degrees of integration compared to the current state of the art [70, 129].

A critical issue aside from material synthesis and fabrication of single processing units, is the integration of the single components into the final neuromorphic structure. The realisation of large, complex and possibly 3D structures incorporating the optically nonlinear materials to achieve functional neuromorphic photonic systems is a challenge. One possibility is the fabrication of small units or unit-agglomerates that can be stitched or assembled together to form the final sail design. Laser and electron beam welding are well known examples for joining thin metal foils but for the above proposed materials may lead to building of stress and defects in the final system. Other options are represented by wafer-bonding techniques widely employed in the semiconductor industry, or the use of nanoscopic bundles of carbon nanotubes or boron nitride nanotubes to tether the processing units together into the final geometry.

The alternative to assembly separate components, is the fabrication of large-scale systems with processing units directly connected into a monolithic artificial neuromorphic network. Different methods can be used for the realisation of micro- and nano-2D and 3D structures $[138,139]$. However, these approaches present several drawbacks including a large aspect ratio with consequent loss of cubic symmetry and difficulties in customising the final design.

Alternatively, techniques such as laser nano-lithography [110, 140] and SPIN [135-137] provide an accessible and controllable approach for the realisation of intricate systems, but due to limited availability of high refractive index photoresists, achieving nonlinearity in systems realised with these methods can be done using inversion techniques [141], and coating methods $[107,108]$.

\section{Conclusions}

Building memristive photonic neuromorphic hardware able to compete and exceed the performance of the von Neuman architectures currently in use is undeniably a very ambitious project, full of challenges and open questions. We are already witnessing increasing efforts to replicate synapses and neurons with photonic platforms and we believe that the neuromorphic photonic field will soon move towards the realisation of fully functional neuromorphic networks. The use of photonic memristive elements will have crucial role in this development, and we believe that rGO will be the preferential candidate to implement these systems. 
The purpose of this Perspective is to offer a positive outlook on the difficulties and the opportunities that a photonic memristive technology could unlock, and to stimulate further discussion and research. The ideas presented here necessitate deeper and more comprehensive analysis, design planning and experimental validation. However, from our considerations emerges that several materials, if combined with proper models and fabrication methods, may allow the design of photonic memristive components to be use in neuromorphic architectures.

\section{Abbreviations}

2D: Two-dimensional; 3D: Three dimensional; Al: Artificial intelligence; CMOS: Complementary metal-oxidesemiconductor; CPU: Central processing unit; DARPA: Defense Advanced Research Projects Agency's; GO: Graphene Oxide; GPU: Graphic processing unit; GST: Ge $\mathrm{Sb}_{2} \mathrm{Te}_{5}$; IPC: Integrated photonic circuit; I-V curves: Current-voltage curves; LIF: Leaky-integrate-and-fire; M: Memristance; OB: Optical bistability; OPCM: Optical phase change material; PCM: Phase change material; rGO: Reduced graphene oxide; SPIN: Super-resolution photoinduction-inhibited nanofabrication; STDP: Spike-timing dependent plasticity; T-P loops: Transmission-incident field power loops

\section{Acknowledgements}

The authors thank all the members of the LAIN Laboratory for the inspiring discussions.

\section{Authors' contributions}

All the authors contribute to the preparation of the manuscript. MG conceived the present idea, QZ performed the calculations reported in Fig. 10 and EG wrote the paper with input from all authors. All authors read and approved the final manuscript.

Funding

The authors received no financial support for the research, authorship, and publication of this article.

\section{Availability of data and materials}

The data that support the findings of this study are available on request from the corresponding author, Min Gu.

\section{Competing interests}

The authors declare that they have no competing interests.

\section{Received: 24 July 2019 Accepted: 4 November 2019}

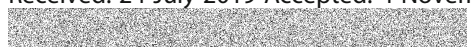

\section{References}

1. McCulloch WS, Pitts W. A logical calculus of the ideas immanent in nervous activity: the bulletin of mathematical biophysics. Bull Math Biophys. 1943;5:115-33.

2. O'Regan G. Artificial intelligence. A Br Hist Comput. 2012:229-52.

3. Intel Corporation. Neuromorphic computing, beyond today's Al. Available at: www.intel.com.au/content/www/au/en/ research/neuromorphic-computing.html. Accessed 12 June 2019.

4. Pedretti $\mathrm{G}$, et al. Memristive neural network for on-line learning and tracking with brain-inspired spike timing dependent plasticity. Sci Rep. 2017;7:05480.

5. An H, Bai K, Yi Y. Advances in memristor neural networks - modeling and applications (ed. Calin Ciufudean); 2018.

6. $\quad \mathrm{An} \mathrm{H}$, et al. "Opportunities and challenges on nanoscale 3D neuromorphic computing system." 2017 IEEE International Symposium on Electromagnetic Compatibility \& Signal/Power Integrity (EMCSI). 2017:416-21.

7. Ielmini D, Wong HP, Article R. In-memory computing with resistive switching devices. Nat Electron. 2018;1:333-43.

8. Silver D, et al. Mastering the game of go with deep neural networks and tree search. Nature. 2016;529:484-8.

9. Mattheij J. Another way of looking at Lee Sedol vs AlphaGo. Hugo. 2016; Available at: jacquesmattheij.com. Accessed 13 June 2019.

10. Luo L. Principles of neurobiology. New York: Taylor \& Francis Group; 2015.

11. Mead C. Neuromorphic electronic systems. Proc IEEE. 1990;78:1629-36.

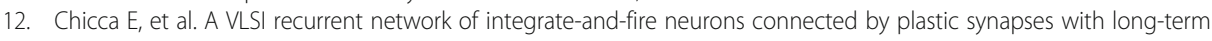
memory. IEEE Trans Neural Netw. 2003;14:1297-307.

13. Jo SH, et al. Nanoscale memristor device as synapse in neuromorphic systems. Nano Lett. 2010;10:1297-301.

14. Tait AN, Nahmias MA, Tian Y, Shastri BJ, Prucnal PR. Nanophotonic information physics: nanointelligence and nanophotonic computing (ed. Naruse, M.). Berlin Heidelberg: Springer; 2014. p. 183-222.

15. Hodgkin AL, Huxley AF. A quantitative description of membrane current and its application to conduction and excitation in nerve. J Physiol. 1952;117:500-44.

16. Stein RB. A theoretical analysis of neuronal variability. Biophys J. 1965;5:173-94.

17. Hebb DO. The organisation of behavior. Berlin, Heidelberg: Wiley/Springer; 1949.

18. Furber S. Large-scale neuromorphic computing systems. J Neural Eng. 2016;13:051001.

19. Le Masson G, Renaud-Le Masson S, Debay D, Bal T. Feedback inhibition controls spike transfer in hybrid thalamic circuits. Nature. 2002;417:854-8.

20. Zou Q, et al. Analog-digital simulations of full conductance-based networks of spiking neurons with spike timing dependent plasticity. Netw Comput Neural Syst. 2006;17:211-33. 
21. Park J, Yu T, Maier C, Joshi S, Cauwenberghs G. Live demonstration: hierarchical address-event routing architecture for reconfigurable large scale neuromorphic systems. In: ISCAS 2012-2012 IEEE Int. Symp Circuits Syst; 2012. p. 707-11.

22. Yu T, Park J, Joshi S, Maier C, Cauwenberghs G. 65K-Neuron integrate-and-fire array transceiver with address-event reconfigurable synaptic routing. In: 2012 IEEE Biomed. Circuits Syst. Conf. Intell. Biomed. Electron. Syst. Better Life Better Environ. BioCAS 2012 - Conf. Publ; 2012. p. 21-4.

23. Brandli C, Muller L, Delbruck T. Real-time, high-speed video decompression using a frame- and event-based DAVIS sensor. In: Proc. - IEEE Int. Symp. Circuits Syst; 2014. p. 686-9.

24. Yang M, Chien CH, Delbruck T, Liu SC. A 0.5 V $55 \mu \mathrm{W} 64 \times 2$ channel binaural silicon cochlea for event-driven stereoaudio sensing. IEEE J Solid State Circuits. 2016;51:2554-69.

25. Indiveri G, Corradi F, Qiao N. Neuromorphic architectures for spiking deep neural networks. Tech Dig - Int Electron Devices Meet IEDM. 2015;15:68-71.

26. Sawada J, et al. A million spiking-neuron integrated circuit with a scalable communication network and interface. Science. 2014;345:668-73.

27. Mostafa H, Müller LK, Indiveri G. An event-based architecture for solving constraint satisfaction problems. Nat Commun. 2015;6:9941.

28. Chicca E, Stefanini F, Bartolozzi C, Indiveri G. Neuromorphic electronic circuits for building autonomous cognitive systems. Proc IEEE. 2014;102:1367-88.

29. Mayr CG, Sheik S, Bartolozzi C, Chicca E. Editorial : synaptic plasticity for neuromorphic systems. Front Neurosci. 2016;10:214

30. Wang Z, et al. Fully memristive neural networks for pattern classification with unsupervised learning. Nat Electron. 2018; $1: 137-45$.

31. Chua L. Memristor-The missing circuit element. IEEE Trans Circuit Theory. 1971;18:507-19.

32. Strukov DB, Snider GS, Stewart DR, Williams RS. The missing memristor found. Nature. 2008;453:80-4.

33. Upadhyaya HM, Chandra S. Polarity-dependent memory switching effects in the Ti/CdxPb 1-xS/Ag system. Semicond Sci Technol. 1995;10:332-8

34. Lau CN, Stewart DR, Williams RS, Bockrath M. Direct observation of nanoscale switching centers in metal/molecule/ metal structures. Nano Lett. 2004:4:569-72.

35. Waser R, Aono M. Nanoionics-based resistive switching memories. Nat Mater. 2007;6:833-40.

36. Pershin $\mathrm{Y}$, Di Ventra M. Spin memristive systems: spin memory effects in semiconductor spintronics. Phys Rev B Condens Matter Mater Phys. 2008;78:5-8.

37. Wu X, et al. Reproducible unipolar resistance switching in stoichiometric ZrO2 films. Appl Phys Lett. 2007;90:11-4.

38. Chua LO, Kang SM. Memristive devices and systems. Proc IEEE. 1976;64:209-23.

39. HP Labs. HP Memristor FAQ. Hewlett-Packard development company, L.P. (2009). Available at: https://www.hpl.hp.com/ news/2008/apr-jun/memristor_faq.html. Accessed: 18 June 2019.

40. Saïghi S, et al. Plasticity in memristive devices for spiking neural networks. Front Neurosci. 2015;9:51.

41. Thomas A, et al. Tunnel junction based memristors as artificial synapses. Front Neurosci. 2015:9:241.

42. Wang Z, Ambrogio S, Balatti S, lelmini D. A 2-transistor/1-resistor artificial synapse capable of communication and stochastic learning in neuromorphic systems. Front Neurosci. 2015;9:438.

43. Chanthbouala A, et al. A ferroelectric memristor. Nat Mater. 2012;11:860.

44. Ohno T, et al. Short-term plasticity and long-term potentiation mimicked in single inorganic synapses. Nat Mater. 2011;10:591.

45. Alibart F, Zamanidoost E, Strukov DB. Pattern classification by memristive crossbar circuits using ex situ and in situ training. Nat Commun. 2013;4:2072.

46. Eryilmaz SB, et al. Brain-like associative learning using a nanoscale non-volatile phase change synaptic device array. Front Neurosci. 2014;8:205.

47. Prezioso M, Hoskins BD, Adam GC, Likharev KK, Strukov DB. Training and operation of an integrated neuromorphic network based on metal-oxide memristors. Nature. 2015;521:62-4.

48. Pickett MD, Medeiros-ribeiro G, Williams RS. A scalable neuristor built with Mott memristors. Nat Mater. 2013;12:10-3.

49. Lim H, et al. Reliability of neuronal information conveyed by unreliable neuristor-based leaky integrate-and-fire neurons: a model study. Sci Rep. 2015:5:9776.

50. Stoliar $\mathrm{P}$, et al. A leaky-integrate-and-fire neuron analog realized with a Mott insulator. Adv Funct Mater. 2017;27: 1604740.

51. Tuma T, Pantazi A, Le Gallo M, Sebastian A, Eleftheriou E. Stochastic phase-change neurons. Nat Nanotechnol. 2016;11:693.

52. Lim H, et al. Relaxation oscillator-realized artificial electronic neurons, their responses, and noise. Nanoscale. 2016;8: 9629-40.

53. Adam GC, Khiat A, Prodromakis T. Challenges hindering memristive neuromorphic hardware from going mainstream. Nat Commun. 2018;9:5267.

54. Ferreira De Lima T, Shastri BJ, Tait AN, Nahmias MA, Prucnal PR. Progress in neuromorphic photonics. Nanophotonics. 2017:6:577-99.

55. Caulfield HJ, Dolev S. Why future supercomputing requires optics. Nat Photonics. 2010;4:261-3.

56. Shastri BJ, et al. Neuromorphic photonics, principles of. Berlin Heidelberg: Springer; 2018.

57. Psaltis D, Farhat N. Optical information processing based on an associative-memory model of neural nets with thresholding and feedback. Opt Lett. 2008;10:98-100.

58. Abu-Mostafa YS, Psaltis D. Optical neural computers. Sci Am. 1987:256:88-95.

59. Jutamulia S, Yu FTS. Overview of the hybrid optical neural networks. Opt Laser Technol. 1996;28:59-72.

60. Hill MT, Frietman EEE, De Waardt H, Khoe GD, Dorren HJS. All fiber-optic neural network using coupled SOA based ring lasers. IEEE Trans Neural Netw. 2002;13:1504-13.

61. Rosenbluth D, Kravtsov K, Fok MP, Prucnal PR. A high performance photonic pulse processing device. Opt Express. 2009;17:22767. 
62. Fok MP, et al. Signal feature recognition based on lightwave neuromorphic signal processing. Opt Lett. 2011;36:19-21.

63. Kravtsov KS, Fok MP, Prucnal PR, Rosenbluth D. Ultrafast all-optical implementation of a leaky integrate-and-fire neuron. Opt Express. 2011;19:2133.

64. Hughes TW, Minkov M, Shi Y, Fan S. Training of photonic neural networks through in situ backpropagation. Optica. 2018:5:864-71.

65. Nahmias MA, Shastri BJ, Ferreira De Lima T, Tait AN. Neuromorphic photonics. Opt Photonics News. 2018;29:36-41.

66. Zhang Q, Yu H, Barbiero M, Wang B, Gu M. Artificial neural networks enabled by nanophotonics. Light Sci Appl. $2019 ; 8: 42$.

67. Fok MP, Tian Y, Rosenbluth D, Prucnal PR. Asynchronous spiking photonic neuron for lightwave neuromorphic signal processing. Opt Lett. 2012;37:3309-11.

68. Gholipour B, et al. Amorphous metal-sulphide microfibers enable photonic synapses for brain-like computing. Adv Opt Mater. 2015;3:635-41.

69. Agnus $\mathrm{G}$, et al. Two-terminal carbon nanotube programmable devices for adaptive architectures. Adv Mater. 2010;22:702-6

70. Cheng Z, Ríos C, Pernice WHP, Wright CD, Bhaskaran H. On-chip photonic synapse. Sci Adv. 2017;3:e1700160.

71. Chakraborty I, Saha G, Sengupta A, Roy K. Toward fast neural computing using all-photonic phase change spiking neurons. Sci Rep. 2018;8:12980.

72. Feldmann J, Youngblood N, Wright CD, Bhaskaran H, Pernice WHP. All-optical spiking neurosynaptic networks with selflearning capabilities. Nature. 2019;569:208-14.

73. Shen Y, Harris NC, Englund D, Soljacic M. Deep learning with coherent nanophotonic circuits. Nat Photonics. 2017;11:441-7.

74. Tait AN, et al. Neuromorphic photonic networks using silicon photonic weight banks. Sci Rep. 2017;7:7430.

75. Koch U, Hoessbacher C, Emboras A, Leuthold J. Optical memristive switches. J Electroceram. 2017;39:239-50.

76. Emboras A, et al. Nanoscale plasmonic memristor with optical readout functionality. Nano Lett. 2013;13:6151-5.

77. Wu H, Zhou J, Lan C, Guo Y, Bi K. Microwave memristive-like nonlinearity in a dielectric metamaterial. Sci Rep. 2014;4:5499.

78. McCall SL. Instabilities in continuous-wave light propagation in absorbing media. Phys Rev A. 1974;9:1515-23.

79. Gibbs HM, McCall SL, Venkatesan TNC. Differential gain and bistability using a sodium-filled fabry-perot interferometer. Phys Rev Lett. 1976;36:1135-8.

80. Sharif MA, Majles Ara MH, Ghafary B, Salmani S, Mohajer S. Experimental observation of low threshold optical bistability in exfoliated graphene with low oxidation degree. Opt Mater (Amst). 2016;53:80-6.

81. Liu Z, et al. Nonlinear optical properties of graphene oxide in nanosecond and picosecond regimes. Appl Phys Lett. 2009;94:21902.

82. Castro Neto AH, Peres NMR, Novoselov KS, Geim AK. The electronic properties of graphene. Rev Mod Phys. 2009;81:109-62.

83. Bonaccorso F, Sun Z, Hasan T, Ferrari AC. Graphene photonics and optoelectronics. Nat Photonics. 2010;4:611-22.

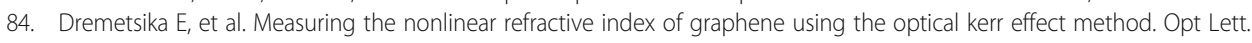
2016;41:3281-4.

85. Peres NMR, Bludov YV, Santos JE, Jauho AP, Vasilevskiy MI. Optical bistability of graphene in the terahertz range. Phys Rev B - Condens Matter Mater Phys. 2014;90:125425.

86. Yao X, Belyanin A. Giant optical nonlinearity of Graphene in a strong magnetic field. Phys Rev Lett. 2012;108:255503.

87. Zhao F, et al. Functionalized graphitic carbon nitride for metal-free, flexible and rewritable nonvolatile memory device via direct laser-writing. Sci Rep. 2014;4:5882.

88. De Silva KKH, Huang HH, Joshi RK, Yoshimura M. Chemical reduction of graphene oxide using green reductants. Carbon N Y. 2017;119:190-9.

89. Zheng X, Jia B, Chen X, Gu M. In situ third-order non-linear responses during laser reduction of graphene oxide thin films towards on-chip non-linear photonic devices. Adv Mater. 2014;26:2699-703.

90. Tian $\mathrm{H}$, et al. Cost-effective, transfer-free, flexible resistive random access memory using laser-scribed reduced graphene oxide patterning technology. Nano Lett. 2014;14:3214-9.

91. Wang Y, Mi H, Zheng Q, Ma Z, Gong S. Graphene/phase change material nanocomposites: light-driven, reversible electrical resistivity regulation via form-stable phase transitions. ACS Appl Mater Interfaces. 2015;7:2641-7.

92. Miller KJ, Haglund RF, Weiss SM. Optical phase change materials in integrated silicon photonic devices: review. Opt Mater Express. 2018;8:2415.

93. Cheng Z, et al. Device-level photonic memories and logic applications. Adv Mater. 2018;30:1802435.

94. Zhang W, Mazzarello R, Wuttig M, Ma E. Designing crystallization in phase-change materials for universal memory and neuro-inspired computing. Nat Rev Mater. 2019;4:150-68.

95. Feldmann J, et al. Calculating with light using a chip-scale all-optical abacus. Nat Commun. 2017;8:1256.

96. Ríos C, et al. Integrated all-photonic non-volatile multi-level memory. Nat Photonics. 2015;9:725-32.

97. Rios $C$, et al. Controlled switching of phase-change materials by evanescent-field coupling in integrated photonics [invited]. Opt Mater Express. 2018;8:2455.

98. Zheng J, et al. GST-on-silicon hybrid nanophotonic integrated circuits: a non-volatile quasi-continuously reprogrammable platform. Opt Mater Express. 2018;8:1551.

99. Liu S, Wei J, Gan F. Optical nonlinear absorption characteristics of crystalline Ge 2Sb2Te5 thin films. J Appl Phys. 2011; 110:33503.

100. Muramoto K, et al. VO2-dispersed glass: a new class of phase change material. Sci Rep. 2018;8:2275

101. Lopez R, Haglund RF, Feldman LC, Boatner LA, Haynes TE. Optical nonlinearities in VO 2 nanoparticles and thin films. Appl Phys Lett. 2004;85:5191-3.

102. Kürüm U, et al. The third order nonlinear optical characteristics of amorphous vanadium oxide thin film. Appl Phys A Mater Sci Process. 2011;104:1025-30.

103. Peng C, Cheng L, Mansuripur M. Experimental and theoretical investigations of laser-induced crystallization and amorphization in phase-change optical recording media. J Appl Phys. 1997;82:4183-91.

104. Sharma N, Sharda S, Katyal SC, Sharma V, Sharma P. Effect of Te on linear and non-linear optical properties of new quaternary Ge-se-Sb-Te chalcogenide glasses. Electron Mater Lett. 2014;10:101-6.

105. Wen S, Meng Y, Jiang M, Wang Y. Multi-level coding-recoding by ultrafast phase transition on Ge2Sb2Te5 thin films. Sci Rep. 2018;8:4979.

106. Wuttig M, Yamada N. Phase-change materials for rewriteable data storage. Nat Mater. 2007;6:824-33. 
107. Goi E, Mashford BS, Cumming BP, Gu M. Tuning the refractive index in gyroid photonic crystals via lead-chalcogenide nanocrystal coating. Adv Opt Mater. 2016;4:226-30.

108. Goi E, Yue Z, Cumming BP, Gu M. A layered-composite nanometric Sb2Te3 material for chiral photonic bandgap engineering. Phys Status Solidi A. 2018;215:1800152

109. Yue Z, Xue G, Liu J, Wang Y, Gu M. Nanometric holograms based on a topological insulator material. Nat Commun. 2017:8:15354.

110. Goi E, Yue Z, Cumming BP, Gu M. Observation of type I photonic Weyl points in optical frequencies. Laser Photonics Rev. 2018;12:1700271

111. Liu S, Wei J, Gan F. Nonlinear absorption of Sb-based phase change materials due to the weakening of the resonant bond. Appl Phys Lett. 2012;100:111903.

112. Liu J, Liu S, Wei J. Origin of the giant optical nonlinearity of Sb2 Te3 phase change materials. Appl Phys Lett. 2010;97: 261903.

113. Leuthold J, Koos C, Freude W. Nonlinear silicon photonics. Nat Photonics. 2010;4:535-44.

114. Nozaki K, et al. Ultralow-power all-optical RAM based on nanocavities. Nat Photonics. 2012;6:248-52.

115. Polyanskiy, M. N. Refractiveindex.info. Available at: https://refractiveindex.info. Accessed: 13 June 2019

116. Schmiedova $V$, et al. Physical properties investigation of reduced graphene oxide thin films prepared by material inkjet printing. J Nanomater. 2017:55:3501903.

117. Kravets VG, et al. Engineering optical properties of a graphene oxide metamaterial assembled in microfluidic channels. Opt Express. 2015;23:1265.

118. Zheng X, Jia B, Chen X, Gu M. Giant optical nonlinear response of graphene oxide films. In: Frontiers in Optics 2013 FW6C.5; 2013.

119. Yue M, Si J, Yan L, Yu Y, Hou X. Enhanced nonlinear optical properties of reduced graphene oxide decorated with silver nanoparticles. Opt Mater Express. 2018;8:698-703.

120. Humlicek J, Lukes F, Schmidt E. Handbook of Optical Constants of Solids (ed. PALIK, E. D.): Academic Press. California; 1998. p. 607-36.

121. Koos C, Jacome L, Poulton C, Leuthold J, Freude W. Nonlinear silicon-on-insulator waveguides for all-optical signal processing. Opt Express. 2007;15:5976-90.

122. Chu CH, et al. Active dielectric metasurface based on phase-change medium. Laser Photonics Rev. 2016;10:986-94.

123. Van Bilzen B, et al. Production of VO2 thin films through post-deposition annealing of V2O3 and VOx films. Thin Solid Films. 2015;591:143-8.

124. Laniel JM, Hô N, Vallée R, Villeneuve A. Nonlinear-refractive-index measurement in As2S3 channel waveguides by asymmetric self-phase modulation. J Opt Soc Am B. 2005;22:437-45.

125. Boguslawski J, Sobon G, Zybala R, Sotor J. Dissipative soliton generation in Er-doped fiber laser mode-locked by Sb2Te3 topological insulator. Opt Lett. 2015;40:2786-9.

126. Wang J, Long Y. On-chip silicon photonic signaling and processing: a review. Sci Bull. 2018;63:1267-310.

127. Ríos C, et al. In-memory computing on a photonic platform. Sci Adv. 2018;5:5759.

128. LeCun Y, Bengio Y, Hinton G. Deep learning. Nature. 2015;521:436-44.

129. Lin X, et al. All-optical machine learning using diffractive deep neural networks. Science. 2018;361:1004-8.

130. Goi E, Gu M. "Laser printing of a nano-imager to perform full optical machine learning," in 2019 Conference on Lasers and Electro-Optics Europe and European Quantum Electronics Conference, OSA Technical Digest. Washington, D.C.: Optical Society of America; 2019. paper jsi_p_3.

131. Gu M, Fang X, Ren H, Goi E. Optically digitalized holography: a perspective for all-optical machine learning. Engineering. 2019;5 $363-65$.

132. Cheng C, Liu K, Xiang B, Suh J, Wu J. Ultra-long, free-standing, single-crystalline vanadium dioxide micro/nanowires grown by simple thermal evaporation. Appl Phys Lett. 2012;100:103111.

133. Hemanadhan M, Bapanayya C, Agarwal SC. Simple flash evaporator for making thin films of compounds. J Vac Sci Technol A. 2010;65:62-626.

134. Miyake $\mathrm{M}$, Chen $\mathrm{Y}-\mathrm{C}$, Braun PV, Wiltzius P. Fabrication of three-dimensional photonic crystals using multibeam interference lithography and electrodeposition. Adv Mater. 2009;21:3012-5.

135. Zheng $X$, et al. Highly efficient and ultra-broadband graphene oxide ultrathin lenses with three-dimensional subwavelength focusing. Nat Commun. 2015;6:8433.

136. Gan Z, Cao Y, Evans R a, Gu M. Three-dimensional deep sub-diffraction optical beam lithography with $9 \mathrm{~nm}$ feature size. Nat Commun. 4:2061, 2013.

137. Cao Y, Gan Z, Jia B, Evans RA, Gu M. High-photosensitive resin for super-resolution direct-laser-writing based on photoinhibited polymerization. Opt Express. 2011;19:19486-94

138. Juodkazis S, et al. Sculpturing of photonic crystals by ion beam lithography: towards complete photonic bandgap at visible wavelengths. Opt Express. 2011;19:5802-10.

139. Qi M, et al. A three-dimensional optical photonic crystal with designed point defects. Nature. 2004;429:538-42.

140. Goi E, Cumming B, Gu M. Impact of cubic symmetry on optical activity of dielectric 8-srs networks. Appl Sci. 2018;8:2104.

141. García-Santamaría F, et al. A germanium inverse woodpile structure with a large photonic band gap. Adv Mater. 2007; 19:1567-70.

\section{Publisher's Note}

Springer Nature remains neutral with regard to jurisdictional claims in published maps and institutional affiliations. 